Mens

revue d'histoire intellectuelle de l'Amérique française

MENS

\title{
L'aventure artistique du peintre de Saint-Denys Garneau
}

\section{Giselle Huot}

Volume 4, numéro 2, printemps 2004

URI : https://id.erudit.org/iderudit/1024597ar

DOI : https://doi.org/10.7202/1024597ar

Aller au sommaire du numéro

\section{Éditeur(s)}

Centre de recherche en civilisation canadienne-française

\section{ISSN}

1492-8647 (imprimé)

1927-9299 (numérique)

Découvrir la revue

Citer cet article

Huot, G. (2004). L'aventure artistique du peintre de Saint-Denys Garneau. Mens, 4(2), 211-271. https://doi.org/10.7202/1024597ar

\section{Résumé de l'article}

De Saint-Denys Garneau (1912-1943) a voulu originellement déployer son œuvre en un diptyque littéraire et pictural. Nous tentons une reconstitution de l'aventure du peintre, de l'éclosion de son désir, en passant par ses études de dessin à l'École des beaux-arts de Montréal (1924-1927) où il côtoie les Palardy, Jori Smith, Borduas et Lemieux. Il retrouve les trois derniers à l'atelier de Holgate où il s'exerce à l'art du nu (1932). Il s'astreint à une formation personnelle - puisque la quête artistique est basée sur la quête ontologique et sur la culture générale - et ambitionne d'être le premier à " renouveler l'art du paysage » et aussi de participer à un mouvement de renaissance artistique en son pays. Il se lie plus tard avec d'autres peintres dont Muhlstock et Lyman et des critiques d'art. Puis, vient l'abandon de la peinture qui suit celle de l'écriture (1937). Sa quête artistique se mue ou encore est englobée dans une aventure spirituelle et religieuse qui a préséance jusqu'à la fin de sa vie. S'il n’a pas eu le temps de développer un style propre contrairement aux autres peintres de sa connaissance, son œuvre picturale n'est pas sans mérite. Parallèlement à l'œuvre du peintre exposée à plusieurs reprises depuis 1993 et reproduite dans deux albums (1993 et 2001) qui illustre surtout le paysagiste, d'autres œuvres sont encore inconnues du public, dont une série de fusains produits aux Beaux-Arts et de nus à l'atelier de Holgate ainsi que des portraits, par celui dont Jean-Paul Lemieux se remémorant sa jeunesse en 1967 rappelle qu'il « dessinait très bien ».
Ce document est protégé par la loi sur le droit d'auteur. L'utilisation des services d’Érudit (y compris la reproduction) est assujettie à sa politique d'utilisation que vous pouvez consulter en ligne.

https://apropos.erudit.org/fr/usagers/politique-dutilisation/ 


\title{
L'AVENTURE ARTISTIQUE DU PEINTRE DE SAINT-DENYS GARNEAU ${ }^{1}$
}

\author{
Giselle Huot \\ Centre de recherche Lionel-Groulx \\ Fondation de Saint-Denys-Garneau
}

\section{Résumé}

De Saint-Denys Garneau (1912-1943) a voulu originellement déployer son œuvre en un diptyque littéraire et pictural. Nous tentons une reconstitution de l'aventure du peintre, de l'éclosion de son désir, en passant par ses études de dessin à l'École des beaux-arts de Montréal (1924-1927) où il côtoie les Palardy, Jori Smith, Borduas et Lemieux. Il retrouve les trois derniers à l'atelier de Holgate où il s'exerce à l'art du nu (1932). Il s'astreint à une formation personnelle - puisque la quête artistique est basée sur la quête ontologique et sur la culture générale - et ambitionne d'être le premier à « renouveler l'art du paysage » et aussi de participer à un mouvement de renaissance artistique en son pays. Il se lie plus tard avec d'autres peintres dont Muhlstock et Lyman et des critiques d'art. Puis, vient l'abandon de la peinture qui suit celle de l'écriture (1937). Sa quête artistique se mue ou encore est englobée dans une aventure spirituelle et religieuse qui a préséance jusqu'à la fin de sa vie. S’il n'a pas eu le temps de développer un style propre contrairement aux autres peintres de sa connaissance, son œuvre picturale n'est pas sans mérite. Parallèlement à l'œuvre du peintre exposée à plusieurs reprises depuis 1993 et reproduite dans deux albums (1993 et 2001) qui illustre surtout le paysagiste, d'autres œuvres sont encore inconnues du public, dont une série de fusains produits aux Beaux-Arts et de nus à l'atelier de Holgate ainsi que des portraits, par celui dont JeanPaul Lemieux se remémorant sa jeunesse en 1967 rappelle qu’il « dessinait très bien ». 


\section{Abstract}

This article examines De Saint-Denys Garneau's (1912-1943) journey as a visual artist. As a young student at Montreal's École des beaux-arts, he had originally hoped to organise his work into a literary and pictorial diptych. During this period, he worked alongside Palardy, Jori Smith, Borduas, and Lemieux, and eventually rendered nudes in Holgate's workshop. Hoping to renew landscape art and to participate in Quebec's artistic renaissance, be linked up with other visual artists, including Mublstock and Lyman, and with a number of art critics. However, shortly after forsaking writing in 1937, be also abandoned painting. At this time, his artistic quest was engulfed by a spiritual and religious journey that would dominate the rest of his all-too-short life. As a result, Garneau was unable to truly develop his own pictorial style. Yet his work is not without artistic merit - artist Jean-Paul Lemieux recalled that Garneau "sketched very well." Even so, and in spite of several exbibits and the recent publication of two art catalogues devoted to his work, which mainly highlight his landscapes, many of his paintings remain unknown to the public. These include a series of charcoal sketches produced while he was at the École des beaux-arts, the nudes painted in Holgate's workshop, and a number of portraits.

Au souffle frais du matin, c'est un peintre qui part en rêve et part en chasse, le pas allègre. Un xil attentif et l'autre en joie... Tout : à droite, à gauche, au-dessus, et d'ici là. C'est un peintre qui promène ce qu'il est parmi ce qu'il y a. Propos sur l'babitation du paysage, $1936^{2}$

L'aventure artistique est celle d'Hector de Saint-Denys Garneau (1912-1943) d'après ses écrits, mais aussi une tentative de recréation de l'atmosphère dans laquelle baignait l'artiste. S'il s'est épanché longuement et profondément sur son aventure intérieure, il n'est pas aussi disert sur l'aventure artistique qu'il partage avec d'autres. Il laisse beaucoup d'ombre sur les gens qu'il a côtoyés, des maîtres et aussi des peintres comme lui en quête de beauté et d'art, ainsi que sur les événements concrets qui ont eu ou pu avoir quelque réper- 
cussion sur son esthétique ou sur son œuvre. Toutefois, l'aventure artistique ne peut être comprise et expliquée sans accorder une grande part à l'aventure ontologique, voire spirituelle ou religieuse, qui en est, du moins en ce qui concerne de SaintDenys Garneau, pour ainsi dire l'alpha et l'oméga. Après avoir présenté cette aventure, nous donnons un aperçu d'une partie de sa production picturale qui n'a pas été reproduite dans les deux albums consacrés à son œuvre ${ }^{3}$.

\section{L'aventure artistique}

\section{1) La gestation de l'artiste : l'enfance (avant 1924)}

"On ne devient pas peintre, on naît peintre », dira plus tard celui qui allait devenir son ami, le peintre Louis Muhlstock. Né à Montréal le 13 juin 1912, de Saint-Denys Garneau ${ }^{4}$ s'en va vivre à Sainte-Catherine de Fossambault en 1916, dans le domaine seigneurial de ses ancêtres maternels Juchereau Duchesnay acquis par sa mère l'année de sa naissance. Très tôt laissé en liberté dans la splendeur de cette nature, cet « enfant est en train de bâtir un village [...] Et qui sait / Tantôt l'univers ${ }^{5} »$. Dès l'âge de cinq ou six ans, il s'affaire à recréer son propre monde, comme nous le rapporte sa mère :

je revois comme si c'était hier notre de St-Denys assis à table avec nous. Il a cinq ou six ans. [...] pendant que nous causons tout en mangeant, lui construit dans son assiette une maison, avec aussi une cheminée ! et un petit chemin pour y conduire, et puis un lac où se promène la sauce. [...] Je revois toujours son regard d'enfant tout absorbé dans son plan qui progresse, sans se rendre le moindrement compte, qu'il est à table pour rendre son repas, non pour dessiner. C'est qu'il dessine à la journée longue 6 . 
Dans le deuxième cahier de son journal, de Saint-Denys Garneau nous apprend que la nature découverte dans ce pays ancestral lui ouvre des horizons insoupçonnés qui façonneront à jamais sa vie : c'est " parmi ces paysages poétiques que s'est formé[e] mon âme, c'est là qu'elle a conçu ces aspirations artistiques, qui dirigent ma volonté plus que toute autre chose. Car le besoin de Beauté, de v[r]aie Beauté pure, plus que l'amour même du devoir, me tient dans la voie du bien. » Il identifie plus spécifiquement la source de ce désir de peindre qui l'habite si tôt :

Ma mère, douée d'une âme très sensible aux beautés de la nature, m'amenait souvent marcher à travers les grands bois de la montagne, vêtus d'un feuillage vert tendre au printemps, ou rougissant, enflammés comme des soleils couchants, dorés, fauves, aux temps d'automne. J'y pris le goût que j'ai pour la peinture?

C'est d'ailleurs à Sainte-Catherine, sollicité par la beauté lunaire, qu'il produit sa première peinture, une aquarelle titrée Lever de lune ${ }^{8}$. Au printemps de 1923, toute la famille revient s'installer à Montréal, dans la maison de Westmount achetée par Madame Garneau en 1913. Cette résidence, pas plus que le Manoir de Sainte-Catherine, n'affiche de tableaux aux murs. Qu'a-t-il donc connu de peinture dans son enfance, à part celles qu'il voit dans les églises ? Quelles connaissances en art possède-t-il avant son arrivée à l'École des beauxarts ? L'on ne sait. Cependant, la première peinture qu'il a pu admirer dans l'entourage familial est le portrait de sa grandmère paternelle Élodie Globensky réalisé par Napoléon Bourassa.

Par sa mère, Hermine Prévost, de Saint-Denys Garneau descend des Juchereau Duchesnay, des seigneurs, des militaires, des hommes politiques, et des Prévost, de riches bourgeois, des militaires aussi. Par son père, Paul Garneau, il ap- 
partient à une lignée d'intellectuels. Peu de Québécois peuvent se targuer d'appartenir à une quatrième génération d'écrivains (par son oncle !). Son arrière-grand-père est l'historien national François-Xavier Garneau (1809-1866), son grandpère, le poète Alfred Garneau (1836-1904), et son oncle, l'historien Hector Garneau (1872-1954), conservateur de la Bibliothèque municipale de Montréal (1916-1930), surtout connu pour ses rééditions de l'Histoire du Canada de FrançoisXavier Garneau.

Dans l'ascendance maternelle de son père, on remarque les Globensky, une famille d'origine polonaise'. Il existe des liens de parenté entre la famille de son arrière-grand-père Léon Globensky et celle de Louis-Joseph Papineau. Leurs épouses respectives, Marguerite-Angélique Limoges et Julie Bruneau, étaient cousines germaines. Les deux familles étaient aussi très liées et la famille de Léon Globensky se rendait souvent l'été à Montebello. Louis-Joseph Papineau appréciait la compagnie des trois sœurs Globensky. Il aurait donné un bijou à Élodie, grand-mère de l'auteur. Il entretint aussi une correspondance avec la sœur de celle-ci, Marie-Louise.

Louis-Joseph Papineau était également ami avec François-Xavier Garneau. C'est à Montebello qu'Élodie Globensky aurait été présentée au fils de l'historien national, Alfred Garneau, alors traducteur en chef au Conseil législatif et poète, qu'elle épouse le 6 octobre 1862. La fille de Louis-Joseph Papineau, Azélie, mariée au peintre Napoléon Bourassa, est l'amie intime d'Élodie Globensky. Napoléon Bourassa a fait un croquis des trois sœurs Globensky et, en 1873, un portrait d'Élodie $^{10}$ aux merveilleux yeux d'or noir, qu'elle semble avoir légués à de Saint-Denys Garneau et à plusieurs autres de ses descendants.

De Saint-Denys Garneau n'a connu ni ses grands-parents maternels (†1895 et 1897) qui ont laissé sa mère totale- 
ment orpheline à l'âge de 12 ans, ni son grand-père paternel, décédé huit ans avant sa naissance, qu'il célébrera dans une conférence en novembre $1927^{11}$. On sait qu'il allait visiter sa grand-mère Élodie qui s'éteint à Montréal le 16 mai 1927, alors qu'il a près de 15 ans et qu'il est toujours à l'École des beaux-arts ${ }^{12}$. Chez elle, il a sûrement admiré son portrait peint par Napoléon Bourassa. Sa grand-mère lui a peut-être parlé qui sait ? - de cet ami, homme de grande culture, initié à la littérature, à la musique et à la peinture, à la fois écrivain, peintre et sculpteur, qui a également conçu les plans de quelques édifices religieux et qui rêvait de mettre sur pied une école des beaux-arts ${ }^{13}$.

2) L'apprenti peintre : l'École des beaux-arts de Montréal (octobre 1924-juin 1927)

Fondée en 1922, sous l'impulsion d'Athanase David, le Secrétaire de la province de Québec, la nouvelle École des beaux-arts de Montréal ouvre ses portes à l'automne de $1923^{14}$. De Saint-Denys Garneau, qui souhaite déjà à cette époque se consacrer à la fois à la littérature et à la peinture ${ }^{15}$, s'y présente le 15 octobre 1924 au "Cours du soir » en "Dessin artistique ». En fin d'année, le lundi 25 mai 1925, sous la présidence de l'Honorable Athanase David, se tient l'inauguration de la deuxième exposition publique des travaux des élèves, qui dure toute la semaine, de même que la deuxième proclamation des prix. De Saint-Denys Garneau gagne le $2^{\mathrm{e}}$ prix, une médaille de bronze pour un sujet imposé : une frise. Son nom est mentionné dans le journal Le Canada ${ }^{16}$. L'année suivante, la fierté générée par ce prix lui fera signer un de ses travaux : de St-Denys Garneau, Artiste-Peintre, couronné par l'Académie Française, $1^{\text {ère }}$ Division.

De Saint-Denys Garneau fréquente l'École à la même époque que les René Chicoine, Fleurimond Constantineau, 
Sylvia Daoust, Madeleine Desrosiers, la future femme de Borduas, Léopold Dufresne, Émile Gauthier, Jean-Marie Gauvreau, Clarice Holland, Miriam Holland, Francisco (Franck) Iacurto, Paul Leroux, Belle Richstone et ceux qu'il retrouvera plus tard après la sortie de l'École, Paul-Émile Borduas, Jean-Paul Lemieux, Jean Palardy et Marjorie (Jori) Smith.

Alors que Palardy semble plutôt satisfait de l'enseignement aux Beaux-Arts, Borduas et Lemieux le décrient ${ }^{17}$. Que pense alors de Saint-Denys Garneau de l'enseignement général à l'École des beaux-arts et de ses professeurs ? Il suit sans doute les cours d'histoire de l'art inaugurés par J.-B. Lagacé le 20 janvier 1925 et peut-être ceux d'Émile Vaillancourt, le titulaire de la nouvelle chaire de l'histoire de l'Art en Canada. Mais il étudie surtout sous la tutelle de l'architecte Albert La Rue (1924-1925), des artistes-peintres Joseph Saint-Charles (1925-1926) et Henri Charpentier (1926-1927). En 1928, il exprime son intention de parler d'eux dans ses mémoires, mais le projet n'est pas allé plus avant ${ }^{18}$. Une dizaine d'années après sa sortie de l'École, à la suite d'une rencontre à une exposition des peintres français, il tiendra Joseph Saint-Charles en bien piètre estime et l'évoquera en ces termes :

M. X., lui, vénérable professeur de dessin d'après chapiteaux antiques (nous voilà en pleine poussière) n'a eu de joie d'aucune sorte. Ni même d'envie, qui est le plus bas tribut qu'on puisse payer à ce qui est admirable. Exactement le silence de la matière en face de l'esprit, de ce qui n'est pas en face de ce qui est. «À ces prix-là, n'importe lequel de nos débutants pourrait faire fortune !» (textuellement). Nous passons une série de bonnes farces ${ }^{19}$.

À la fin de 1935, dans son texte "À propos de BeauxArts ", qu'il destinait à $L a$ Relève, mais qui n'aura qu'une des- 
tination posthume, il s'exprime en termes sévères sur l'enseignement des arts, critiquant l'académisme de l'École et le manque d'originalité et de liberté, s'insurgeant contre les arts décoratifs que favorisa Charles Maillard, le directeur de l'École de 1925 à 1945. Déplorant la pauvreté des œuvres artistiques, il en fait porter une partie du blâme sur l'enseignement déficient des beaux-arts, sur son manque de réalisme dont il identifie les deux malfaçons, l'académisme " outré » et la trop grande part accordée à l'art décoratif :

D'abord, académisme outré qui ne cultive pas les éléments personnels de l'élève, et ne lui apprend pas à découvrir sa vision, et qui ne sait pas éliminer ceux qui ne sont pas susceptibles de progrès, qui ne possèdent pas les aptitudes requises, non de la main, mais de l'âme. Ensuite, la trop grande part que prend l'art décoratif dans les cours de peinture et de dessin. Familiariser le jeune avec des formes parfaites qu'il ne comprend pas au lieu de formes vivantes qu'il sentirait mieux, et qui lui permettraient de regarder avec ses propres yeux, cela paraît manquer de logique. À la rigueur, on pourrait alterner les deux. Ne pas initier les jeunes à la couleur, qui est bien ce qui les frappe davantage, et non pas la ligne, qui est une abstraction. [...] Nous touchons ici à ce que nous croyons la seconde erreur de notre système d'enseignement en art : la trop grande part accordée à la décoration. Après une discipline étroite où l'élève fut par trop contraint, en tout cas mal éveillé, on le lance dans ce domaine où l'imagination est mầtresse. Ce qui arrive, c'est qu'il tombe dans l'arbitraire. Il peut bien se conformer à des règles extérieures de composition et de coloris, mais ce n'est pas là qu'il peut acquérir le sens profond de l'ordonnance et de l'harmonie ${ }^{20}$.

Pleinement conscient " qu'une discipline est nécessaire », il remarque toutefois que « le sens de la discipline est 
une éducation de la liberté, ce qui suppose à la base une prise de conscience de la liberté ». De plus, le rôle véritable de l'éducateur devrait être non celui d'« un empêcheur d'erreur ", mais d'un véritable " éveilleur de vérité ». Il ne minimise pas les difficultés auxquelles sont confrontés les éducateurs en arts, mais il n'en préconise pas moins la nécessité d'une évolution :

Je sais avec quels difficiles problèmes sont aux prises ceux qui s'occupent de cette éducation artistique, et que certains mettent à leur tâche une grande sincérité et toute leur ardeur. Je sais qu'ils se rattachent à une tradition longuement élaborée, et que leur doctrine est celle de bien des milieux conservateurs en Europe. Je sais aussi qu'ils tâchent sans cesse à améliorer leurs méthodes. Mais notre époque, qui remet en question toutes les valeurs, toutes les institutions, veut avant tout le retour à la vie, à la réalité vivante, quitte ensuite à conserver les formes encore viables de la tradition. Elle entend se rattacher aux œuvres du passé, à sa sève même, et non aux formules qu'il nous légua toutes faites. Les éducateurs devraient prendre les devants sur cette route du renouveau ; sans quoi ils seront bientôt forcés de suivre ou de disparaittre.

Cette évolution est d'autant plus nécessaire que les problèmes reliés à la stagnation de l'enseignement artistique ont des conséquences néfastes sur toute la vie nationale :

Si j'ai insisté sur ce point, c'est que là se centre toute l'éducation, c'est que ce problème, sa solution a de grandes répercussions sur toute la vie, sur la vie nationale en particulier, et que ce problème est éminemment actuel et que tous ceux qui tiennent à la survie du peuple canadien-français doivent se dévouer à sa solution dans le sens de la vie profonde, de la réalité véritable ${ }^{21}$. 
De Saint-Denys Garneau n'a jamais, à notre connaissance, entretenu de correspondance avec ses professeurs aux Beaux-Arts, comme il le fait avec ses professeurs laïcs et jésuites. Ses professeurs de dessin semblent n'avoir été que cela, des professeurs, qui ne seront jamais pour lui des maîtres. Cette vie d'études plus libre s'achève et avec elle la fin de ses études à l'École des beaux-arts. La reprise de ses cours en septembre 1927 au collège Sainte-Marie ${ }^{22}$ ne lui laisse plus de loisirs suffisants pour les poursuivre.

3) Le peintre en devenir : études et formation personnelle (été 1927 biver 1932)

Tout son apprentissage à l'École des beaux-arts ne serait rien ou si peu si de Saint-Denys Garneau n'avait poursuivi ses études générales et fait œuvre de formation personnelle. Pour lui, la quête artistique est basée sur la quête ontologique, qui embrasse l'aventure spirituelle ou religieuse, et sur la culture générale.

La jeunesse adopte souvent des principes ou des idées qui deviennent les guides ou éclaireurs de toute une vie. Dès l'âge de 17 ans, de Saint-Denys Garneau a déjà décidé de l'orientation qu'il entend donner à sa vie. En octobre 1929, à la fin d'une page de journal, qui lui tient aussi lieu de devoir de collège, il affirme : "mon intention est de me vouer à l'art : à la poésie et à la peinture ${ }^{23}$ ». C'est qu'il a « éprouvé dès [s]on jeune âge cette nécessité de rejoindre la réalité, de la posséder ailleurs, plus parfaitement, éternellement ${ }^{24}$ ». Cette quête de la beauté l'entraîne inévitablement vers le bien : «ces aspirations artistiques [...] dirigent ma volonté plus que toute autre chose. Car le besoin de Beauté, de vraie Beauté pure, plus que l'amour même du devoir, me tient dans la voie du bien $^{25}$.» 
Ce désir de beauté l'aiguillonne à nourrir de grandes ambitions, qui sont presque sans limites en sa prime jeunesse. À l'âge de 17 ans, il se risque à dévoiler à sa correspondante préférée d'alors, qui avait fouetté sa fierté en le traitant de "sans-ambition ", l'objet même de son ambition. Il vient d'écrire sa première critique d'art sur la peinture canadienne ${ }^{26}$, et s'il note que c'est « dans la peinture régionaliste qu'on réussit le mieux », qu'il y a " de jolis talents, des conceptions intéressantes de la nature canadienne toujours ", il constate qu'il n'y a cependant

rien qui frappe et qui retienne par la profondeur de la pensée, du sentiment, par l'envergure de la conception. Il n'y a rien de classique, je veux dire qui s'attache à rendre l'homme universel. Il n'y a pas d'œuvres symboliques qui s'efforcent de rendre par des attitudes, par des compositions, par des paysages animés de sentiment, de grands états d'âme. Il n'y a pas d'art dramatique, rien d'absolument personnel qui tienne de l'homme et qui ne soit pas seulement une copie de la réalité, de la nature, idéalisée si vous voulez, mais une copie toujours. L'art du paysage a une grande prospérité. Il n'y a pas de " créations » qui vivent de la grandeur de la passion.

Ce sont ses forces et ses visions qu'il veut mobiliser pour tenter de contrer cette pauvreté :

Tout reste donc à faire de ce côté. Il y a au Canada un bel avenir en peinture, non au point de vue réussite matérielle, mais au point de vue d'art, de gloire. Celui qui viendra le premier apporter ce qui manque, la passion des grandes et belles visions, celui-là sera le premier et devra, ce me semble, en récolter beaucoup de gloire tôt ou tard. 
Je voudrais que ce fût moi. J'y tâcherai, advienne que pourra.

Dites maintenant que je n'ai pas d'ambition ${ }^{27}$ !

Parce que les poètes et les artistes jouissent d'un statut privilégié de par ce pouvoir qui leur a été conféré de découvrir des beautés non immédiatement accessibles au commun des mortels, ils ont le devoir d'en rendre compte :

Les poètes et les artistes sont des privilégiés qui voient des beautés qui ont besoin d'être montrées aux autres pour que ceux-ci les aperçoivent. Or l'artiste fait une vie vide, une vie ratée, s'il garde pour lui ces aperçus sur la Beauté de Dieu, s'il la savoure et meurt avec, dans son cœur qu'elle consume, l'égoïste ! Non! Le but de l'artiste, c'est de faire voir au monde la Beauté qu'il voit. Il a sa part de responsabilités, une grande part. Son travail est un devoir. Dieu lui demandera compte de ce qu'il a montré aux hommes des choses qu'll lui a fait voir ${ }^{28}$.

À cet impérieux devoir d'œuvrer se jouxte celui de faire œuvre belle qui commande à son tour d'autres devoirs. Les dons naturels ne suffisent pas, un travail acharné est nécessaire pour se montrer digne de cette vocation et pour engendrer « de grandes et belles visions ${ }^{29} »:$ " je travaille, je travaillerai jusqu'au bout et je deviendrai ce que je pourrai atteindre de mieux [...] Je travaille [...] et je peine sans relâche dans le dur chemin qui mène vers la Beautét ${ }^{30}$.»

Très tôt le fantaisiste se forge en entreprenant un véritable travail de formation. Non seulement il entend faire œuvre d'art, mais vouer sa vie à l'art implique qu'il se conçoive et se veuille œuvre d'art ${ }^{31}$. S'il n'aimerait rien de mieux que de se consacrer tout entier au plus tôt à ses occupations de prédilection, il se rend compte de la nécessité d'un apprentissage qui reste encore à venir : 
Quand j'aurai fini mon cours d'études que je terminerai certainement car la formation des dernières années est la plus importante, quand j'aurai terminé mon cours, dis-je, je m'en irai étudier la peinture en Europe. Je m'en reviendrai ensuite ici et je me donnerai entièrement à la peinture et à la littérature. [...] Il me faut l'inspiration pour que je croie à mon étoile. Je manque de confiance en moi-même. Il faudra de grandes choses pour que je sois sûr de moi. Et je ne ferai de grandes choses que si je travaille. En avant donc! Ce qui me fatigue le plus, c'est de n'être pas capable de me donner entièrement aux arts, c'est d'étudier de petites choses. Mais je comprends que cela est nécessaire et je m'efforce de maitriser mes chimères jusqu'à ce qu'il soit temps de les laisser aller à tous leurs élans ${ }^{32}$.

Dès 1927, il se fait une nécessité de faire partie de cercles littéraires ${ }^{33}$. Toutes ces démarches sont d'autant nécessaires que son but dépasse de beaucoup ses ambitions personnelles qu'il entend faire servir à une noble cause, celle de participer à un mouvement de renaissance, de croissance intellectuelle et artistique :

Je fais partie d'un cercle littéraire de jeunes gens. Cela m'intéresse fort. N'est-ce pas la meilleure façon de former, non seulement des écrivains, mais même une élite qui soit capable de comprendre nos artistes, de créer une atmosphère plus favorable aux arts, d'en propager le goût ? C'est la seule façon, croyons-nous d'étendre le goût des choses intellectuelles, la culture générale. C'est un idéal. Et comme un idéal est un rêve qui doit se réaliser dans un futur parfois éloigné, nous ne nous attendons pas de le voir réalisé immédiatement. Mais c'est par des efforts collectifs et individuels nombreux, par l'initiative et le travail des jeunes que nous parviendrons à faire du Canada un pays intellectuel, où subsiste et prospère la vieille et féconde tradition gréco-latine. 
Les difficultés sont grandes, mais l'important n'est-il pas d'avoir un grand but à atteindre, et d'en montrer le chemin ? Notre peuple jeune et enthousiaste quand il sera convaincu de la grandeur de ce but et de la nécessité de son existence pour la subsistance de notre race canadienne-française, fournira l'effort pour l'atteindre et continuera en grand ce que nous aurons commencé en petit $^{34}$.

Dans ce but, il continuera à travailler sur soi et à se sculpter littéralement au moyen de lectures, de conférences, d'expositions de peinture, de séances de musique, de spectacles, de théâtre, de cinéma, de concerts.

\section{4) Le peintre parmi les peintres (biver 1932-printemps 1937)}

Tant qu'il est au collège, les loisirs sont comptés pour peindre, sauf pendant les petites vacances d'hiver, les vacances d'été, les jours de congé et les congés de maladie, beaucoup plus longs et nombreux que pour la moyenne des collégiens. Que ses congés de maladie lui sont doux! Pourvu, bien sûr, qu'ils lui laissent le loisir de se livrer à l'écriture et à la peinture.

En février 1932, il doit abandonner ses études pour ne les reprendre qu'en septembre suivant. À l'automne de 1933, alors qu'il est en première année de Philosophie au collège Sainte-Marie, les médecins, lui ayant découvert une lésion au cœur contractée à la suite d'une attaque de fièvre rhumatismale à l'âge de seize ans ${ }^{35}$, lui recommandent d'interrompre ses études. De Saint-Denys Garneau se retrouve avec beaucoup de temps libre pour vaquer à ses occupations favorites. Les médecins ont évidemment recommandé du repos, ce qu'il interprète assez librement et subjectivement pour les inclure à haute dose dans sa convalescence. Il possède toujours ce désir de se "donner à créer un art qui exprime notre nature ». 
La tâche est titanesque pour celui à qui on vient d'annoncer qu'il a « une maladie de cœur»: "Je rirais, si ce n'était que je n'ai pas du tout envie de rire : je me sens écrasé par la tâche que je voudrais accomplir. " Faut-il pour cela déjà abdiquer avant même d'avoir vraiment commencé ? "Il n'est rien d'aussi désolant que l'impuissance. Mais aussi, il n'est rien de si méprisable que l'acceptation de l'impuissance, par avance, sans avoir essayé ses forces. Je veux tenter fortune. » Est-ce une prémonition ? Un doute s'infiltre en lui et il se surprend à entrevoir la possibilité d'un échec quant à la réalisation de ce rêve :

Si je subis un échec complet, ce n'est pas le courage qui m'aura manqué ce sera la puissance de mon cœur qui aura fait défaut à ses désirs. Cela doit briser étrangement que de se manquer ainsi à soi-même. C'en est assez pour écraser du coup tout orgueil. Cela aura du moins cela de bon ${ }^{36}$.

Il tient bon. Son rêve de 1930 a mûri, s'est précisé, et ayant pu mieux constater l'indigence du milieu culturel, il se rappelle au combat : " À nous la faute, à nous le devoir de réparer la faute, de réveiller le peuple, de le secouer. Il n'est que temps. " Deux buts concomitants, l'un, plus immédiat, qui prépare le second, lointain, qui le continue et l'épanouit : " voici mon but : me former une pensée vaste, juste et vigoureuse, bien établie, un goût sûr ; me réaliser dans l'art ", afin de pouvoir rejoindre ce désir d'influer sur son milieu :

Voilà donc mon but : créer de la beauté, et participer à un mouvement de renaissance au Canada. Non pas faire des chefs-d'œuvre ; je connais mes limites, mais me réaliser à la limite de mon possible, être un de ceux qui agissent vers la beauté, être un facteur de formation pour le goût, ici, et un facteur d'élévation dans la solidarité du monde. 
Voilà mon but, mon idéal. Il est haut placé, un peu abstrait, si tu veux. Mais il n'est pas sans tenir compte de la réalité, il y est au contraire solidement appuyé ; sur toutes les réalités, depuis la réalité idéale et éloignée jusqu'à la réalité physique, pratique ${ }^{37}$.

S'il n'affectionne pas particulièrement les contraintes, il reconnaît par ailleurs qu'il est disposé pour son art à s'imposer des sacrifices qu'il ne ferait pas autrement :

Cependant que mon art m'élève, que j'y satisfais mon amour de la beauté, de la forme [...] que cela exalte le meilleur de moi ; que je consens, pour être moralement beau et créer de la beauté, beaucoup plus de sacrifices que je ne ferais dans la vie pratique. $\mathrm{Si}$ je laissais prendre la première place à la réalité pratique, je perdrais beaucoup en propreté morale. Il faut que l'étoile qui me guide soit haute et lointaine ${ }^{38}$.

Cette période, surtout à partir de 1934, est celle pendant laquelle se forme véritablement son esthétique nourrie par ses lectures de livres d'art ${ }^{39}$, par les nombreuses expositions de peinture qu'il visite, à plusieurs reprises pour certaines, esthétique éprouvée au contact des peintres qu'il connaît et des milieux qu'ils fréquentent. Si l'œuvre est conçue et élaborée dans la solitude, l'échange des idées est nécessaire pour celui qui a toujours l'intention de contribuer à influencer et à élever quelque peu le milieu culturel canadien-français. Autant il juge essentiels à sa formation les cercles littéraires, autant il se doit de fréquenter les cercles ou les ateliers des peintres. Il renoue alors de façon plus soutenue avec les peintres qu'il connaissait depuis l'École des beaux-arts et fait d'autres belles rencontres qui le font pénétrer dans le cénacle d'artistes très connus à l'époque.

Les deux peintres avec lesquels de Saint-Denys Garneau gardera des contacts amicaux après les Beaux-Arts sont 
Jean Palardy ${ }^{40}$ et Marjorie (Jori) Smith, devenus mari et femme en 1930. Il ira faire un atelier de peinture avec eux dans les Laurentides en mai-juin $1932^{41}$. Il les retrouvera en août 1933 en Charlevoix où il est allé peindre et où les Palardy ont élu domicile ${ }^{42}$.

Peintre et graveur, Edwin $\mathrm{H}$. Holgate ${ }^{43}$ enseigne la gravure sur bois à l'École des beaux-arts de Montréal à partir de l'année 1926-1927 jusqu'en 1934, mais de Saint-Denys Garneau n'y suit pas ses cours. Dans son texte " $\grave{A}$ propos de Beaux-Arts », de Saint-Denys Garneau mentionne : "J'aurai peut-être l'occasion de parler un jour de l'effort d'Edwin Holgate vers une formation plus libre et plus profonde des jeunes aspirants à l'art ${ }^{44}$. » Projet auquel il n'a pas donné suite, malheureusement, car il fait partie de ces « jeunes aspirants à l'art » qui ont bénéficié de l'enseignement du maître.

En 1928, Holgate possède un grand atelier au 364, rue Dorchester Ouest (l'actuel René-Lévesque). Outre ses cours aux Beaux-Arts, il " décide $\mathrm{d}[\mathrm{e}]$ tenir le soir des séances de pose pour les étudiants qui veulent s'exercer à l'art du nu. Il devient vite l'un des professeurs les plus populaires de l'école ${ }^{45} »$. Jean-Paul Lemieux, qui fréquente aussi l'atelier de Holgate, partage l'opinion de De Saint-Denys Garneau sur lui et dira :

À vrai dire, le seul que je respectais était Edwin Holgate. Il avait un grand atelier près de Burnside et il y donnait des classes de modèle vivant. J'avais une grande confiance en Holgate. J'admirais en lui le peintre aussi bien que le professeur. Je l'ai rencontré aux Beaux-Arts où il enseignait la gravure sur bois. Plusieurs élèves venaient à son atelier. En fait, un d'entre eux s'est fait connaitre comme écrivain, Saint-Denys Garneau. [...] Holgate était vraiment un professeur célèbre. Tout le monde voulait aller chez lui. Il était un artisan très exigeant et merveilleux ${ }^{46}$. 
En quelle année de Saint-Denys Garneau y est-il allé ? S'il a bien fréquenté l'atelier de la rue Dorchester, alors c'est entre 1928 et 1932, puisque c'est cette année, dit-on, que Holgate déménage maison et studio sous un même toit au 3535 de l'avenue Lorne. D'autre part, on dit que Lemieux fréquente cet atelier dans la première moitié des années trente. En ce qui concerne de Saint-Denys Garneau, nous croyons que ce ne peut être qu'à l'hiver et au printemps de 1932. Pour cette période, nous n'avons que peu de points de repère sur ses activités. Cependant, dans sa lettre du 24 février 1932 écrite à Montréal, il confie : "J'ai abandonné mon année, quitte à la recommencer l'an prochain. Ma santé ne me permet pas de continuer cette année. J'ai donc beaucoup de temps à moi, lequel temps j'emploie à lire, écrire et surtout peindre et dessiner. Je suis très convenablement actif $f^{47}$. » De Saint-Denys Garneau a dessiné une série de nus féminins, dont nous parlerons en deuxième partie, que nous croyons avoir été réalisés lors de ces séances au studio de Holgate.

Peut-être de Saint-Denys Garneau connaissait-il JeanPaul Lemieux avant même l'École des beaux-arts puisque qu'ils ont tous deux fréquenté le collège Loyola à la même époque ? Ce qui est sûr, c'est qu'ils se revoient à l'atelier de Holgate :

dans son studio situé 364, rue Dorchester Ouest, Holgate tient, le soir, des séances de pose pour ceux qui veulent s'exercer à l'art du nu. Lemieux y dessine régulièrement dans la première moitié des années trente développant son individualité créatrice; il faut citer aussi Paul-Émile Borduas, Jori Smith, Goodridge Roberts, Stanley Cosgrove et le jeune poète québécois SaintDenys Garneau que Lemieux avait déjà connu à l'École des beaux-arts de Montréal et dont la recherche artistique déborde l'écriture pour englober ponctuellement la pratique de la peinture ${ }^{48}$. 
En 1965, en évoquant ses compagnons de l'atelier Holgate, Lemieux dira : "En fait, un d'entre eux s'est fait connaittre comme écrivain, Saint-Denys Garneau. Il dessinait très bien. Nous sortions ensemble après les cours pour prendre un café $^{49}$. »

Paul-Émile Borduas était arrivé à l'École des beauxarts, mieux préparé que la plupart des élèves puisqu'il avait été pendant deux ans l'apprenti d'Ozias Leduc. Après sa sortie de l'École, c'est aussi à l'atelier de Holgate, donc en 1932, que de Saint-Denys Garneau retrouve Borduas. Aux artistes déjà mentionnés qui fréquentent l'atelier de Holgate, une autre source ajoute le nom du $\mathrm{D}^{\mathrm{r}}$ Norman Bethune ${ }^{50}$. À peu près à cette époque, Borduas se lie avec les amis de La Relève, principalement avec Robert Élie, qui publiera le premier volume à lui être consacré, l'année même de la mort de De SaintDenys Garneau ${ }^{51}$.

En décembre 1935, dans sa "Chronique des BeauxArts ", publiée dans La Relève, de Saint-Denys Garneau écrit : «Toutefois, ceux qui sont allés au salon d'automne n'ont pas perdu leur temps. Car ce à quoi je me suis arrêté ici, je ne m'y suis guère arrêté au salon, mais plutôt devant les dessins de Louis Muhlstock ${ }^{52}$, devant les tableaux d'Edwin Holgate, et quelques autres. Le reste, on l'oubliait ${ }^{53}$. " Sur la relation de De Saint-Denys Garneau avec Louis Muhlstock nous en connaissons un peu plus que sur celles avec les autres peintres. En compagnie de Robert Élie, son compagnon de prédilection pour les visites des expositions, de Saint-Denys Garneau se rend voir les œuvres de Muhlstock ${ }^{54}$ exposées pour la première fois en solo, du 16 octobre au 27 novembre 1935 à l'Art Association, la Galerie des Arts (l'actuel Musée des beauxarts). Pendant qu'il entreprend de lui consacrer entièrement sa deuxième critique d'art pour La Relève $e^{55}$, Robert Élie se fait l'interprète des deux amis pour solliciter une rencontre avec 
le peintre ${ }^{56}$. C'est le début d'une amitié puisque les deux amis, écrit Muhlstock :

m'invitèrent à leur tour à participer, chez eux, à de merveilleuses sessions d'audition de musique.

Au cours de ces rencontres, tenues le samedi soir ou le dimanche après-midi, $j$ 'eus le plaisir de rencontrer leurs amis intimes : Jean Le Moyne, Gertrude Hodge, François Rinfret et Claude Hurtubise, quoique je connus moins ce dernier.

Nous écoutions la musique dans un silence absolu. Du fromage, des biscuits, des pâtisseries et des petites tasses de café étaient servis, puis la discussion s'engageait. À l'époque, ma connaissance du français était suffisante pour me permettre de converser avec eux. Mais au mieux, j'étais un bon auditeur. Je n'étais pas en mesure de participer aux échanges portant, par exemple, sur la littérature, la philosophie ou la religion. Mon plaisir était de partager leur compagnie et d'écouter. La musique, l'art, la philosophie et la religion étaient leurs domaines de prédilection. Ils en avaient une connaissance approfondie et une grande compréhension. J'étais le seul peintre à temps plein au sein du groupe $\mathrm{e}^{57}$.

Contrairement à Palardy, Jori Smith, Lemieux et Borduas, en présence desquels il peignait ouvertement, de Saint-Denys Garneau a caché pendant longtemps à Muhlstock son travail intensif en peinture : "Je ne découvris que bien plus tard que de Saint-Denys était aussi un peintre. Je ne sais pourquoi, mais à aucun moment au cours de ces après-midi ou de ces soirées, ni ses amis ni de Saint-Denys n'évoquèrent en ma présence le fait qu'il peignît ${ }^{58}$. " Cela a été pour Muhlstock un constant sujet d'étonnement, jusqu'à la fin de sa vie. Un jour, de Saint-Denys Garneau a enfin osé lui avouer son terrible secret, allant même jusqu'à lui faire voir quelques-unes de ses créations : 
Aussi, fut-ce avec grand plaisir que j'examinai plusieurs peintures qu'il vint me montrer à mon atelier, après un séjour qu'il avait fait à la Trappe d'Oka. Ceci me donna l'occasion de manifester mon appréciation. Plus tard, de Saint-Denys m'envoya aussi une carte sur laquelle il avait fait reproduire un paysage qu'il avait peint. Il percevait avec sensibilité les paysages qui se transposaient naturellement sur la toile. Ces derniers étaient bien composés et ils avaient été pleinement observés et ressentis - ils présentaient une couleur juste et une atmosphère. On sentait qu'il avait aimé les peindre ${ }^{59}$.

Par la suite, un témoignage de l'auteur à ce sujet situe cette rencontre à l'été de 1936. À une correspondante amie, de Saint-Denys Garneau confie :

J'ai vu Muhlstock l'autre jour. Il a beaucoup travaillé cet été et nous a montré des choses très intéressantes. De la peinture surtout. Une grande abondance de lumière et de mouvement. De la belle forme aussi [...] Je lui ai montré quelques-unes de mes toiles de cet été. Il a trouvé cela intéressant, surtout du point de vue de la couleur. Cela m'encourage, et je continue à travailler de pied ferme ${ }^{60}$.

Il y a sans doute eu échange de toiles entre eux. Est-ce à ce moment ? Ne reste de cet échange que ce mot de Louis Muhlstock, écrit sur du papier brun d'emballage, peut-être à l'arrière d'une toile qu'il aurait offerte à de Saint-Denys Garneau : "Fair exchange is no robbery ${ }^{61}$. " " Robbery », fait sans doute référence à la réaction de notre auteur-peintre qui, considérant Muhlstock comme un peintre infiniment supérieur à lui, ne trouvait pas l'échange équitable. Louis, le fidèle, se souviendra toujours avec amitié et nostalgie de son ami de Saint-Denys Garneau, l'évoquant à chacune de nos rencontres. Pour mieux commémorer sa mémoire, il a offert à la Fondation de Saint-Denys-Garneau, dont il était membre du Co- 
mité d'honneur depuis sa création en 1994, de financer un prix littéraire en son honneur ${ }^{62}$.

De Saint-Denys Garneau n'a pu connaître John Lyman qu'à partir de 1931, lors du retour définitif au pays de ce grand voyageur qui a vécu longtemps en Europe et beaucoup voyagé de par le monde, mais sans doute plusieurs années plus tard. En 1937, il visite des expositions où sont présentées des œuvres de Lyman, en particulier celle qui réunit onze peintres à l'Arts Club et une exposition solo à la Galerie Scott. Lyman enseigne et aime s'entourer de jeunes peintres des deux communautés linguistiques. Il leur ouvre les portes de sa demeure, où il reçoit des peintres connus, dont Biéler et Holgate, et des membres influents du milieu des arts. "C'est là que l'on discuta l'idée de mettre sur pied des associations artistiques ${ }^{63}$. Ces soirées fournissaient aussi une occasion aux francophones et aux anglophones de se rencontrer, alors que de jeunes artistes y trouvaient encouragement et soutien ${ }^{64}$. » À cette époque, dans certains ateliers, on croit que "la culture nuit à l'expression artistique en interposant des réminiscences et des éléments hétérogènes entre le créateur et son œuvre ». Ce qui n'est évidemment pas le cas de Lyman, "esprit distingué et cultivé, il joint à son talent de peintre l'intelligence des problèmes plastiques et la sûreté de goût d'un critique judicieux et averti ${ }^{65} \%$.

Nous pouvons supposer que de Saint-Denys Garneau apprécie chez Lyman l'intellectuel de vaste culture, le critique d'art reconnu qui travaille à faire connaître l'art vivant et qui voudra plus tard écrire en français un livre sur son maître et ami Morrice ${ }^{66}$. De plus, comme de Saint-Denys Garneau, Lyman accorde énormément d'importance au regard et bien que reconnu comme intellectuel, il se glorifie de " ne vivre que par les yeux ", formule qui deviendra le titre du film qui lui sera consacré : Je vis par les yeux ${ }^{67}$. 
La seule mention par de Saint-Denys Garneau de sa fréquentation de la résidence de Lyman se situe au printemps de 1937, après la parution de son recueil et au moment où la crise a refait surface : "Puis mon aventure chez Lyman. Je n'existais pas, je n'avais rien à dire : donc ce que j'avais pu dire était mensonge. Mon livre et ma critique sur les peintres français. J'ai étalé là, dans une humiliation incommensurable, une misère dérisoire et horrible. Mélange de tragique, de méprisable et de ridicule ${ }^{68}$. » En avril 1938, de Saint-Denys Garneau évoque dans son journal quelques réflexions d'un groupe de peintres sur l'art. Il est dommage qu'il ne mentionne ni le lieu où se déroule cette discussion ni les noms des peintres qu'il cite. Toutefois, il n'est pas impossible que cette rencontre ait eu lieu chez Lyman ${ }^{69}$.

Bien que relativement peu connu, Lyman a été un peintre qui a laissé sa marque sur l'évolution de la peinture canadienne : «John Lyman fut une des personnalités importantes à prôner l'internationalisme et à répandre des idées qui devaient déclencher des changements artistiques majeurs, non seulement dans les années trente mais pendant les deux décennies suivantes $^{70}$."

De Saint-Denys Garneau se lie également avec des critiques d'art, dont certains sont des peintres, comme Lyman, ou des littérateurs, comme son ami Robert Élie, Henri Girard, Charles Doyon, Émile-Charles Hamel, Paul Dumas et Rodolphe Dubé (alias François Hertel).

$\grave{A}$ remarquer que tous les peintres dont de Saint-Denys Garneau recherche la compagnie et l'enseignement sont ceux qui échappent à l'influence du Groupe des Sept - quoique Holgate y ait appartenu brièvement - pour être davantage attirés par la peinture moderne et la peinture européenne, dont l'École de Paris. Ils ont tous étudié ou voyagé en Europe, ce qui n'était pratiquement que le lot des peintres prometteurs à 
l'époque. Les critiques qu'il fréquente sont aussi sensibles à cette forme d'art et accueillent les nouveautés.

C'est pendant cette période que de Saint-Denys Garneau écrit le plus et le mieux sur sa conception de l'art, sur sa démarche artistique. À part les textes plus structurés, il y a des notes partout. Lorsqu'il examine certaines de ses toiles, il "y découvre un ordre intime plus profond que celui » qu'il y a « mis par travail conscient, consciencieux ». Il en déduit qu'en ce qui le concerne, lui qui se croit " peu déterminé, peu profondément informé ", il y a là " grande part de hasard », alors que pour les grands artistes, il s'agit d'une « détermination intérieure ». Cette détermination est ce qu'il appelle « sa vision ", " sa valeur ontologique », aussi " son subconscient par rapport à l'ordre conscient qu'il y met, à la possession qu'il en a par l'ordre, lequel est proprement de l'art ${ }^{71} »$.

$\mathrm{La}$ vision « qui réalise le rapport spirituel entre le spectacle et le spectateur nous offre le spectateur comme lieu de ce rapport ». C'est que chaque artiste " porte en soi des nécessités qui conditionnent son œuvre", à commencer par "le désir de peindre » : «le regard s'est mis en route pour voir, pour rejoindre le monde », mais cela n'est pas inné. "On ne nait pas avec une vision mais avec la possibilité d'une vision, et en tant qu'artiste, avec le besoin d'une vision, d'une vision harmonieuse du monde. » Cette vision peut être conditionnée par « la technique, l'influence de la dominante d'un paysage, l'influence des maitres, etc. Tout ce qu'il n'apporte pas en naissant. Il y a en outre en lui un principe personnel par quoi il choisit, qui nécessite son choix parmi tout cela ${ }^{72}$. »

Il raconte une expérience de peinture. «Ce paysage constitue une occasion pour ma vision de se réaliser et à la fois une perfection, comme réalisable, de ma vision. » L'expérience se déroule en trois lieux d'habitation. D'abord, relevant du domaine de l'objectivité, «ce paysage extérieur où ma vision 
s'étend et s'établit reine. [...] Pour le faire valoir, l'améliorer, l'interpréter, mais non le changer. Ma vision habite donc ce paysage sous un certain rapport, en tant que l'informant. » $\mathrm{Ce}$ pendant,

par cette prise de possession elle le ramène à l'intérieur de moi où l'élément constant passe de l'objectif au subjectif, et où le regard passe du paysage habité à l'habitation du paysage, au mode d'habitation. Ceci n'est pas successif mais marque une interaction, et comme les divers points où peut porter l'attention. Le paysage donné me sollicite; en tant qu'informé il est sollicité par moi : j'ai pour lui une sollicitude.

Voici l'espace que j'habite. Je m'y meus avec liberté et ma mesure lui est imposée, est imposée à sa grandeur, ma façon de parcourir l'étendue et de rejoindre l'horizon, de m'orienter, d'accrocher les points cardinaux, et de pratiquer entre les nuages des passages pour la lumière.

Enfin, le troisième point d'habitation, qui est « l'emplacement sur la toile pour l'habitation du nouveau venu »:

Là sera le fruit de l'union et le corps devra contenir sensiblement l'âme. C'est à ce moment qu'entre en action tout moi-même, et tant de hasards, et l'intelligence qui surveille pour reconnaitre et le sens critique pour dire : «Ça n'est pas cela. » J'enlève petit à petit tout le voile du papier blanc. Sous ma vigilance la juste réalisation de ma vision dans le paysage s'opère. Et, à la fois, sous mon action se fait l'incarnation de cela sur la toile. Je suis entre ma toile et moi par ma main et mon métier, entre le paysage et moi par ma vision. Et tout à l'heure je figure sur la toile les points matériels qui jalonnent ma vision dans le paysage et tout à l'heure le lien même entre ces points, qui est ma vision ${ }^{73}$. 
Ce peintre toujours habité du même désir d'agir sur son milieu, est déterminé à œuvrer, mais individuellement. Il reconnait qu'il n'entre pas dans ses attributs de militer, de se tourner vers l'action au moyen de mouvements tels les JeuneCanada (1932-1938). Plusieurs de ses amis ou connaissances, qui avaient pour maittre Lionel Groulx, l'avaient créé ou en faisaient partie, dont Robert Charbonneau, Pierre Dansereau, Gilbert Manseau, Jean-Louis Dorais, GeorgesÉtienne Cartier et André Laurendeau. Il peut partager certaines de leurs idées tout en les exprimant différemment. Au lieu de l'action directe il opte, pour être conséquent avec ses aptitudes, à travailler à donner à son pays un artiste qui en relèvera le niveau de culture, comme il l'explique à André Laurendeau :

nos idées, tu sais, sont encore moins dissemblables que tu ne crois. Il est vrai que mes amis, ou plutôt l'un d'eux, Jean Le Moyne, n'a pas du tout confiance en l'action nationale; mais je ne m'entends pas avec lui sur ce point. Au contraire, le problème canadien-français m'intéresse beaucoup et a toujours une certaine part dans mes préoccupations. Seulement, alors que toi tu t'y donnes directement, activement, que tu en fais dès maintenant une occupation et un but, le but de ta vie, je le mets au second plan dans la mienne. Et c'est là une direction de tempérament, n'est-ce pas, beaucoup plus que d'idées. Tu aimes l'action ; moi, je suis plus contemplatif, pour employer un joli grand mot. Si j'étais fait pour l'action, c'est très probablement de ce côté que je me dirigerais. Mais, je le sens bien, j’y ai bien réfléchi, je ne suis pas disposé en ce sens. En me cultivant, en me perfectionnant, je ne perds pas de vue le point de vue national de culture et de mouvement intellectuel, de milieu à créer et d'élan à donner. Surtout plus tard, je pourrai être utile. Mais mes buts sont plus 
éloignés, mes moyens [in]directs. Mais, chacun selon soi, n'est-ce pas $^{74}$ ?

En fait, s'il est sensible au problème canadien-français, il n'accepte pas, comme il l'écrira plus tard dans ses « Notes sur le nationalisme ${ }^{75}$ ", qu'on envisage la culture sous un angle nationaliste. La culture étant « essentiellement humaniste » veut faire « des hommes avec des C[anadiens] F[rançais] et non pas des C[anadiens] F[rançais] avec des hommes ", ce qui lui fait évidemment rejeter l'éducation dite " nationale ». Quelques mois après sa lettre à André Laurendeau, en janvier 1934, il définit plus précisément l'orientation qu'il entend donner à sa vie, comme nous l'avons vu : "créer de la beauté, et participer à un mouvement de renaissance au Canada [...] être un de ceux qui agissent vers la beauté, être un facteur de formation pour le goût, ici, et un facteur d'élévation dans la solidarité du monde ${ }^{76} »$.

À Jean Le Moyne qui lui fera remarquer plus tard qu'il accorde trop d'importance à ce qu'il nomme sa " mission ", il rétorque que sa mission n'est pas que de faire, mais de faire bellement, du mieux possible :

À propos de moi artiste. Je ne crois pas que je donne trop de part à ma « mission ». Je considère simplement cela comme quelque chose que j'ai à faire, comme un autre qui a des dispositions pour la politique se dirige dans la vie politique afin de bien y faire ce qu'il a à y faire, réaliser là le bien qu'il lui est donné de compren$\mathrm{dre}^{77}$.

Il est important pour de Saint-Denys Garneau de tout considérer "dans son ordre ». D'ailleurs, c'est là l'une des qualités ou nécessités du poète : "Chaque chose, si nous la comprenions dans son ordre, serait belle pour nous. Le poète est celui qui voit les choses dans leur ordre. La beauté du fumier qui engraisse la terre dans son ordre, etc. ${ }^{78}$ » Dans cette 
perspective, le paysan est tout aussi important que le poète ou l'artiste, chacun veillant à œuvrer en fonction de ses aptitudes et chacun admirant le travail de l'autre ${ }^{79}$.

Même si ses ambitions ne se concrétisaient pas, tout ne serait pas perdu, puisque la quête de la beauté porte en ellemême sa propre justification : « $\mathrm{Si}$ je ne fais jamais rien, j'aurai toujours la consolation d'avoir tâché vers la beauté, d'en avoir fait la préoccupation de ma vie ${ }^{80}$. $\gg$ Cependant tout est mis en œuvre pour les réaliser. À l'été de 1936, il prévoit exposer une toile au Salon du Printemps de 1937 et il travaille avec ferveur à ses poèmes. En janvier 1937, alors qu'il prépare son recueil, il envisage de s'offrir un voyage d'études et de découvertes en Europe d'une durée minimale d'un an. Cette phase optimiste lui fait même mijoter le projet de présenter Regards et Jeux dans l'Espace au prix David:

il est très probable que j'irai en Europe pour un an, j'espère, et peut-être plus. [...] tu imagines quelle fête cela sera pour moi. [...] Si la plaquette de vers que j’ai l'intention de présenter au prix David remporte quelque succès (tout est possible !) cela facilitera beaucoup les choses pour moi ${ }^{81}$.

Le printemps de 1937 s'annonce prometteur au regard des objectifs qu'il s'est fixés.

\section{5) Le peintre en désenchantement (printemps 1937-1943)}

Cette période n'en est pas tant une de formation que de transformation et de remise en question. Sa vocation d'artiste s'engage irrévocablement dans une voie qu'il n'avait pas prévue. Pourtant le printemps avait si bien commencé ! Avec la parution de son recueil Regards et Jeux dans l'Espace si amoureusement architecturé et si soigneusement mis en page par lui à l'CEuvre de Presse Dominicaine ${ }^{82}$. Avec sa toile Ciel en 
automne qui fait partie de l'exposition du $54^{\mathrm{e}}$ Salon annuel du Printemps à la Galerie des Arts ${ }^{83}$.

La crise qui s'était manifestée bien avant cette grande rampe de lancement, dès 1935 et plus intensément à l'été de 1936, puis qui lui avait laissé quelque répit, le temps d'être porté sur une crête qui lui avait permis ces rebondissements, réapparait plus violente encore aux abords du printemps 1937. Elle mènera de Saint-Denys Garneau au retrait de son recueil quelques semaines après sa parution et lui fera abandonner progressivement l'idée de publications subséquentes. Contrairement à ce qu'on a souvent avancé, ce retrait n'est en aucune façon causé par la critique de Valdombre-Grignon-Grognon ${ }^{84}$, à qui c'est vraiment faire trop d'honneur, mais par la crise intérieure qui s'installe de plus en plus profondément en lui. Hanté par "l'angoisse d'être découvert ", il croit son œuvre entachée d'un manque d'authenticité. C'est parce qu'il s'intente un procès d'imposture, procès où il est à la fois juge et partie, qu'il se fait une obligation de délaisser l'écriture : «On n'a pas droit de jouer par les mots de ce qui ne comporte pas en nous de substance profonde ${ }^{85} \%$. Il se sent acculé à renoncer à exprimer cette beauté qui reste inextricablement liée à la vérité, conditionnée par la vérité : "Je sépare de moins en moins vérité et beauté. Ce qui n'est pas vrai est d'une beauté factice, donc non pas vraiment beau ${ }^{86}$. »

C'est pendant cette période qu'il qualifie de " dépression nerveuse » ou de " neurasthénie » qu'il s'embarque pour ce voyage en Europe qu'il désirait depuis des années et qui devait être le couronnement de son apprentissage de l'art. Tous les peintres qu'il affectionne et qu'il a côtoyés y ont séjourné et presque tous ses amis y sont allés, certains plusieurs fois. Cette « fête » d'au moins un an qu'il se promettait encore en janvier se métamorphose en une "saison en enfer ", qu'il 
abrège à quelques semaines. Parti de Montréal le 2 juillet 1937, il rentre à Québec le 31 juillet ${ }^{87}$.

À son retour, brisé, il s'installe au manoir de SainteCatherine, qu'il ne quittera plus que pour de brèves périodes, jusqu'à sa mort. C'est pour lui le début de la fin de l'aventure artistique telle qu'il la concevait depuis sa jeune adolescence, en un diptyque littéraire et pictural. À part sa correspondance, les seules notes écrites pendant son voyage en Europe que nous lui connaissons commencent ainsi : «Art — Faire surgir, éclairer le subconscient par la découverte du monde. Offrir sa lumière au monde et à Dieu. " Mais un peu plus loin, une réflexion annonce la route dans laquelle il va bientôt s'engager :

Une sorte d'histoire de la pauvreté du monde. En cette époque où les yeux sont si tournés vers la pauvreté matérielle, il est peut-être utile de faire voir la pauvreté réelle intérieure, ontologique, et de faire sentir que le remède n'en est pas dans la politique et l'économique, et qu'il y aura toujours des pauvres parmi nous. Remède ultime : le désert. Esprit de pauvreté ${ }^{88}$.

S'il continue de peindre, son regard se fait cependant de plus en plus virulent sur son œuvre picturale et celui qui, quelques années auparavant, se sentait pratiquement du génie, de l'exaltation lorsqu'il peignait s'engage sur la pente du désenchantement :

J'ai peint hier après-midi. Je vois mon talent dans une plus juste proportion. Un œil assez simple. Parfois de jolies rencontres. C'est tout. Pas un talent créateur. Pas la force d'originalité ni d'exaltation. Donner sa vie à cela ? C'est bien pauvre, c'est tout à fait insuffisant. Je n'ai rien de particulier à dire. Je peux parler joliment de temps en temps ; ça n'a aucune importance ${ }^{89}$. 
Le mois suivant, sa confiance est encore plus ébranlée, et le trouve en train d'évoquer l'orgueil et le mensonge invoqués déjà pour se persuader de renoncer à l'écriture :

J'ai découvert le sens de ce que j'appelais « mon art » et par là le ridicule où j'étais de lui accorder tant d'importance. Qualité de sensibilité, mais non tempérament vraiment créateur. J'ai peint quelque peu dernièrement. Ça n'a pas d'importance : de jolies rencontres au cours de la reproduction de la nature. Dépouiller cela, l'organiser pour en faire une construction vraiment forte : effort épuisant et temps perdu.

Pour en arriver à la même conclusion : «Et si l'on s'y livre avec avidité : disproportion, orgueil et mensonge ${ }^{90}$. "

Celui qui avait écrit " On a fait un grand pas dans la connaissance d'un homme quand on connait la question qui s'offre à la base de cette conscience " et "Seuls vivent ceux qui poursuivent la solution d'une question ${ }^{91}$ " constate avec effroi, au printemps de 1938 : " J'ai perdu en chemin le sens même de la question que je croyais qui m'était posée ${ }^{92} »$. Réfléchissant qu' un " homme est mort quand tout lui semble résolu ", il en vient à découvrir une autre réponse ou un autre modus vivendi : " $\grave{A}$ moin[s] qu'il ne s'absorbe dans la connaissance d'une réponse acceptée : le mystique ${ }^{93}$.»

Le problème auquel il est de plus en plus confronté lui qui a toujours dit et pensé que toute chose était belle dans son ordre - est celui de la destinée humaine, de la sienne, de ce qu'il croit être chez lui la démesure de l'orgueil qui refuse sa réalité pour en créer une autre, mensongère, au moyen de toutes sortes de compromissions. C'est cela qui génère sa crise et le problème récurrent qui consiste à ne pouvoir s'accepter tel qu'il est ou tel qu'il croit être :

Seulement, voici le point. Tout acte, dans le sens le plus large, est une confrontation. C'est une mesure que, en 
la donnant à ce qui est informe, l'on se donne de soimême. Et c'est en ce point que l'orgueil intervient et à sa suite se déclenchent toutes les puissances de refus et de mensonge. Coûte que coûte, au moyen de tous les artifices, tenir à cette mesure, et refuser que cette mesure soit petite. Qu'elle soit grande coûte que coûte par une prostitution à toutes les magies, jusqu'à la noire. $\mathrm{Ne}$ pas se contenter de la mesure qui nous est donnée, de ce qu'on est fait, mais se vouloir plus grand, se vouloir infini, ne serait-ce que par cette exaspération et ce refus, par où d'ailleurs la grenouille creva ${ }^{94}$.

Pour se mettre à la recherche de la perfection, de l'infini, il croit qu'il lui faut réapprendre le vrai chemin qui est de délaisser « l'avarice » pour « la générosité » car « la montagne de l'orgueil se trouve réduite au désert du refus ». Sa " destinée d'ensemble » serait dans une démarche plus humble, de s'abandonner, de se perdre pour se sauver, comme il est dit dans l'Évangile, mais il croit perdre lui-même cette connaissance lorsque lui " apparaît la moindre destinée partielle » qui, dans son cas, est celle de l'art. Après avoir affirmé que « le saint est la plus belle œuvre d'art ", il ajoute que "seul importe le surnaturel, le "besoin de grandeur" par l'humilité, le bonheur qui nous dépasse, le seul possible », cependant que "l'art, en tant que faire, reste essentiellement terrestre », bien qu' " il comporte comme toute ouvre humaine l'infinité du désir ${ }^{95} »$.

Un drame préparé secrètement et longuement, nourri de mille doutes et de tout autant de douleurs qui paraissent sans fin, se fera accompagnement jusqu'à la fin de sa vie, pour vivre ce qu'il nomme une vocation de pauvreté ou du désert, à la recherche de cette authenticité qu'il se refuse tant de fois mais dont il est le plus fidèle garant. Un jour, il se rend compte que malgré rêves et sueurs il n'atteindra jamais le but ou le grand peintre de ses désirs. Il s'astreint à reconnaître ses limites qu'il repousse à l'extrême. La quête ontologique se pour- 
suit, s'intensifie dans une aventure spirituelle et religieuse qui a toujours été sous-jacente à sa démarche artistique, mais qui prendra de plus en plus de place jusqu'à n'être plus que la seule quête importante. En délaissant l'aventure artistique pour l'aventure religieuse, de Saint-Denys Garneau ne délaisse pas l'art, puisque, a-t-il écrit, " le saint est la plus belle œuvre d'art ", rejoignant en cette expression-désir le vœu de sa jeunesse de se "faire œuvre d'art ».

On a parlé de mort artistique bien avant sa mort physique. Cela est juste quant à sa production littéraire et artistique telle qu'il la concevait depuis l'adolescence. Mais jamais, jusqu'à la fin de sa vie, il ne pourra s'empêcher d'être artiste dans l'âme et dans l'œil. Robert Élie a bien relevé cette nécessité irrépressible du « faire » chez de Saint-Denys Garneau, alors même qu'il renonce à l'écriture et, d'une certaine façon, à la peinture : " Tu as toujours fai $[t]$, même lorsque tu n'écrivais pas, c'est justement cela qui caractérise ton attitude devant les belles et les bonnes choses; cette vocation à faire dès que tu es un artiste ${ }^{96}$. "Son être muselé, encarcané par les interdits qu'il érige, allait forcément s'en libérer partiellement.

Pendant les dernières années de sa vie, alors qu'il ne fréquente plus de peintres, il contribue à en former un. Antoine Prévost est son cousin, le fils de son oncle préféré SaintDenys Prévost. S’il ne connait pas encore le poète, il a découvert le peintre, "un "grand" qui comme nous, "les petits", s'occupait à faire des images ». De Saint-Denys Garneau qui, jusqu'à la fin de sa vie conservera ce désir d'éduquer ceux qu'il en juge dignes dans la voie de la beauté, se fera professeur ou tout au moins conseiller pour guider ses jeunes cousins dans leurs efforts picturaux :

Plus tard, à l'occasion, nous eûmes droit à des leçons d'aquarelle, comme à l'hiver de 1942, où de Saint-Denys nous guidait dans la préparation de cartes de sou- 
haits alors que, papa étant malade, tante Mimi nous avait reçus, les enfants, au manoir, pour les fêtes de Noël ${ }^{97}$.

Continuant à faire, mais différemment, comme l'atteste sa production artistique de la dernière période, il s'initie à une autre forme d'art, à partir de 1940 surtout. Il se servira, de ruineuse façon - c'est lui qui l'affirme - de la photographie pour tenter de fixer des scènes de paysages. Il cultive également la beauté en se faisant l'architecte paysager des jardins du manoir, son œil de peintre lui reste qui ne cesse d'y recréer des formes ainsi qu'une profusion harmonieuse de couleurs. Il continue d'habiter le paysage, car la nature et ses beautés sont restées ses ancres nécessaires jusqu'à la fin de sa vie. Si «Dieu a donné la nature à l'homme pour s'en servir », il l'a offerte au poète ou au peintre " pour qu'il l'élève à la dignité de compagne ${ }^{98} »$.

Borduas a aussi vécu une longue période de solitude, de presque absolu silence et d'apparente stérilité. Alors que de Saint-Denys-Garneau « venait de publier ses poèmes [...] Borduas se détourna de la voie commune, si bien pavée de bonnes intentions, pour interroger ce qui était pour lui le total inconnu ${ }^{99}$ ». Robert Élie, qui est à la fois un grand ami de De Saint-Denys Garneau et de Borduas - à l'instar de Maurice Hébert, père d'Anne Hébert, qui avait demandé à Félix-Antoine Savard de rencontrer son cousin de Saint-Denys Garneau à la fin de sa vie ${ }^{100}$ —, voulait mettre en face à face ce dernier et Borduas :

Quand j'ai rencontré Borduas, - (c'est assez profond, je pense comme circonstance) - c'est qu'à ce moment-là justement, j'étais l'ami de Saint-Denys-Garneau, un autre créateur. Saint-Denys-Garneau était réduit au silence, c'était avant qu'il ne meure. Je me souviens d'avoir tenté d'organiser une rencontre entre Borduas 
et Saint-Denys-Garneau. Saint-Denys-Garneau ne pouvait plus correspondre à ce moment-là. Pour moi, c'était chez l'un et chez l'autre, chez l'un qui venait de mourir, cette même tentative d'absolu. Alors Borduas se trouvait juste au début, lui... Il était au début lui de tout ce qu'il a tenté d'impossible. Puis, je le voyais justement se mesurer après de longues années de silence. Il y avait eu 10 années de silence chez Borduas, où il avait rongé son frein et tout à coup, il trouve sa matière, il trouve son milieu et il se lance avec rage même, mais avec aussi une joie secrète qui faisait tout éclater ${ }^{101}$.

À peu près au moment où de Saint-Denys Garneau arrête de peindre, Borduas fait en 1942, à 36 ans, une rentrée fracassante sur la scène des Arts, qui allait le lancer irrévocablement. En 1943, de Saint-Denys Garneau meurt à 31 ans.

Un an après le Refus global, Robert Élie écrit encore :

Les préférences de Borduas vont aux poètes visionnaires de la littérature, de la musique et de la peinture, à ceux-là qui n'ont jamais eu leur place dans la société. Pourtant, il croit à la valeur permanente de leur témoignage. Aussi ne juge-t-il pas vaine la lutte qu'ils ont soutenue avec la société (le monde, dirait un chrétien) et peut-être accorde-t-il à leurs souffrances une valeur rédemptrice. N'y a-t-il rien de commun entre ces poètes et les saints, entre l'homme de désir et l'homme de charitét ${ }^{102}$ ?

\section{La production artistique}

La production artistique garnélienne se divise en deux grandes catégories, la critique d'art et la production picturale. Critique d'art, de Saint-Denys Garneau est aussi critique littéraire et critique musical génial :

Sur la peinture, de Renoir à Cézane, sur la musique, de

Borodine à Schubert, de Mozart à Beethoven, de Bach 
à Debussy, personne au Canada français n'a écrit des notes plus intelligentes, plus subtiles, plus intensément personnelles ${ }^{103}$.

Faute d'espace, nous devons omettre ici la partie sur la critique d'art, qui a le mérite d'avoir été publiée, afin de nous concentrer sur une partie de la production picturale qui n'a pas été reproduite dans les deux albums parus. Les œuvres mentionnées ci-après, sauf pour quelques-unes appartenant au Fonds Hector-de-Saint-Denys-Garneau LMS-0207 de Bibliothèque et Archives Canada, proviennent de collections privées. La plus importante comporte une série de fusains, tous les dessins retrouvés, semble-t-il, produits à l'École des beaux-arts de 1924 à 1927 ; elle regroupe également la plus grande partie d'une série de nus, des fusains et quelques pastels, qui ont été réalisés, croyons-nous, à l'atelier de Holgate, probablement en 1932. En outre, d'autres fusains, des portraits, de la même collection appartiennent aussi à la troisième période $^{104}$. Ces dessins nous font connaître le dessinateur, une autre dimension de l'artiste que nous connaissons surtout comme paysagiste par les deux albums parus. Jean-Paul Lemieux se souvient encore trente ans après l'avoir fréquenté que de Saint-Denys Garneau « dessinait très bien », comme nous l'avons mentionné plus haut.

\section{1) La gestation de l'artiste : l'enfance (avant 1924)}

Les auvres retrouvées de cette période sont peu nombreuses, toutes des aquarelles de petit format. De la première, Lever de lune (1920), il ne reste qu'une photographie ${ }^{105}$; deux autres représentent une scène de campagne et un village aux moulins à vent (ca 1921), alors que la dernière illustre un menu d'anniversaire (ca 1923) ${ }^{106}$. 


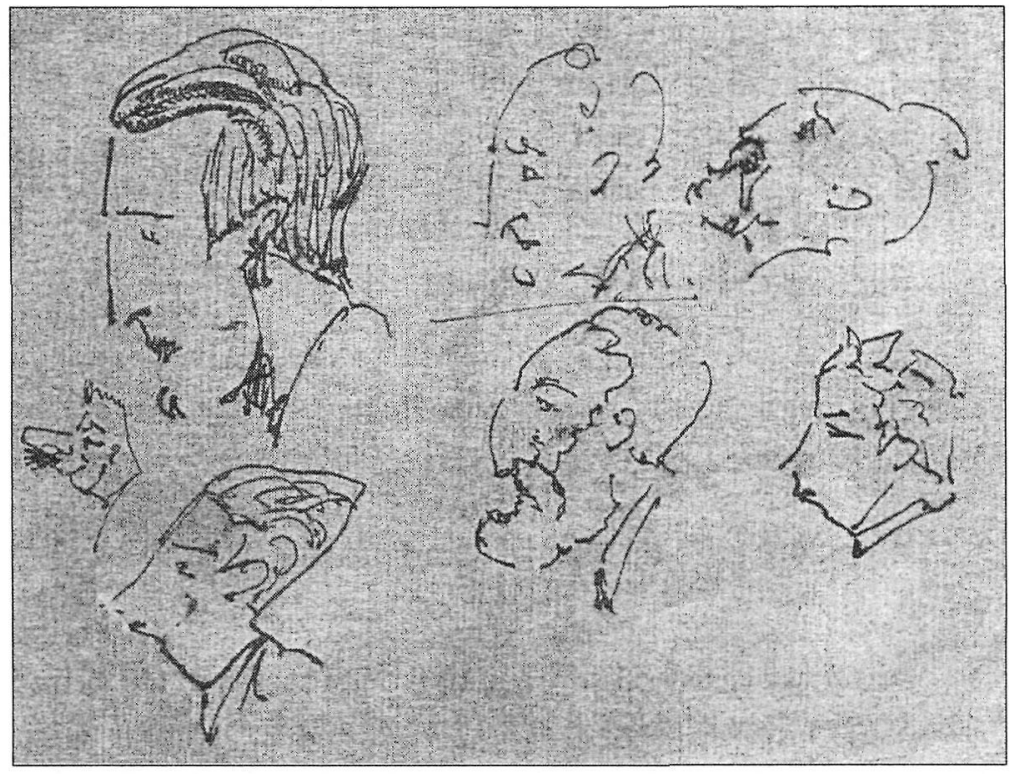

De Saint-Denys Garneau, [Sans titre. Croquis de treize profils]. Détail. Encre (dix) et mine de plomb (trois) sur la couverture d'un bloc de papier à lettres. 25 x 20,2 cm. [1930-1935]. Collection G. H. Anciennement coll. Benoît Lacroix. 
2) L'apprenti peintre : l'École des beaux-arts de Montréal (octobre 1924-juin 1927)

Une vingtaine de fusains de grande dimension, environ $63 \times 48 \mathrm{~cm}$, divisés en trois sections, pour chacune des années d'étude aux Beaux-Arts. Ces dessins sont des sujets imposés, généralement d'après des animaux empaillés ou des plâtres antiques, sauf pour la troisième année, d'après des modèles vivants.

a) 1924-1925 Cours du soir : Dessin artistique. Cours élémentaire (Hommes), $2^{\mathrm{e}}$ Division

Une [Frise] qui n'est peut-être pas celle avec laquelle l'auteur a gagné le deuxième prix et médaille de bronze en mai $1925^{107}$.

b) 1925-1926 Cours du soir : Dessin artistique. Cours moyen (Hommes), 1 ère Division

Neuf pièces représentant des dessins d'animaux, une [Tête de cheval, un [Ibis ou héron et faucon], le premier beaucoup mieux réussi. Puis la représentation humaine avec une [Tête léonine], un [Profil du Moïse de Michel-Ange], une [Tête d'enfant], l'étude du corps humain avec [Nu féminin, sans tête et aux membres tronqués], qu'il signe : de St-Denys Garneau, Artiste-Peintre, couronné par l'Académie Française, $1^{i r e}$ Division, un [Buste féminin sur socle avec figure de profil, aux cheveux torsadés], une [Jeune fille, vêtue de long, aux cheveux dénoués].

En 1925, un [Masque grotesque], qu'il est fort intéressant de comparer à celui de Paul-Émile Borduas, dessiné la même année. Celui de Borduas est évidemment supérieur ; nonobstant le talent, Borduas est aussi plus âgé que de Saint-Denys Garneau de sept ans et il a l'avantage d'avoir été l'apprenti d'Ozias Leduc durant deux ans avant son arrivée aux BeauxArts $^{108}$. 


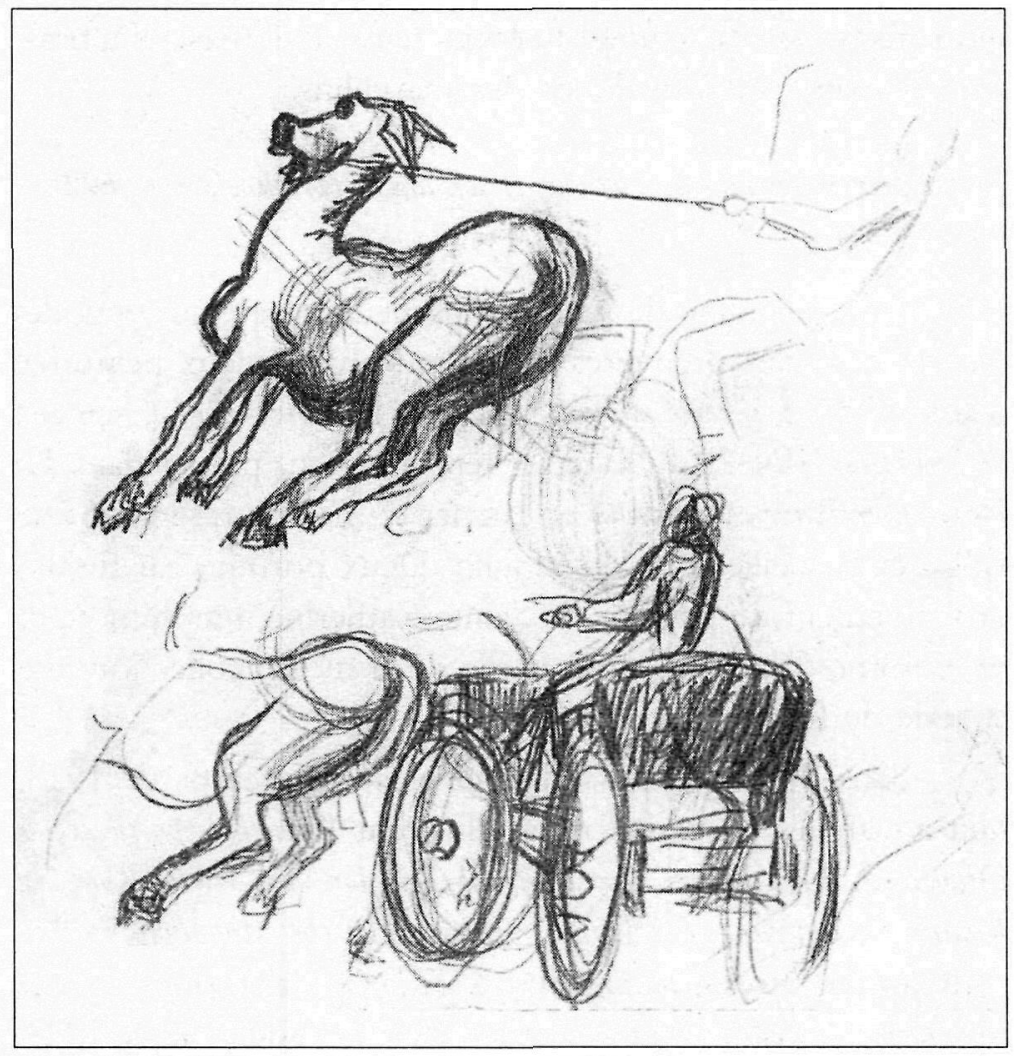

De Saint-Denys Garneau, [Sans titre. Études : cheval, carriole, hommes]. Détail. Mine de plomb sur papier. 20,3 x 24,9 cm. Non signé. Non daté [vers 1930]. Collection G. H. Anciennement coll. Benoît Lacroix. 
c) 1926-1927 Cours du soir : Dessin artistique. Cours supérieur (Hommes)

En troisième année, dix pièces. Tous les sujets sont humains, squelette, études du corps féminin et masculin, bras, jambes, torse, tête, quelques nus masculins.

3) Le peintre en devenir : études et formation personnelle (été 1927 biver 1932)

Une vingtaine de pièces appartiennent à cette période, qui est celle des premières huiles retrouvées, deux peintures d'août 1927, Le vieux moulin et La roue du vieux moulin, signées de St-D.G. ${ }^{109}$. De 1928, les deux reproductions parues dans La Revue scientifique et artistique en janvier et mai, représentant une église et la vieille roue du moulin. Deux portraits au fusain, probablement de paysans de Sainte-Catherine, une femme et un homme d'âge mûr, font partie de la même collection que la série de fusains des Beaux-Arts.

Sans doute peintes à l'hiver et au printemps de 1928, huit aquarelles de petit format illustrent Recueil de poésies : Le Dinosaure, L'Automne, La chute de Rawdon, La vieille Roue du Moulin, L'automne, La Lune, Aimer et In the Moonlight; elles sont toutes titrées, et signées de St.D.G., sauf pour In The Moonlight, signée de St.D. Garneau. Une autre aquarelle de 1928, signée aussi des initiales, représente un paysage illustrant une carte à Françoise Charest. Enfin, L'île d'en haut, très beau pastel sec sur papier ${ }^{110}$.

De 1930, quatre pièces datées et deux autres de la même époque, dont l'une, à la mine de plomb sur papier, représente des études, cheval, carriole, hommes, et l'autre, fusain sur papier, un cheval de trait. Deux aquarelles représentent, l'une un paysage champêtre ornant une carte de vœux à Françoise Charest, l'autre [La vieille "chanfferie »] près du Manoir, qu'il 


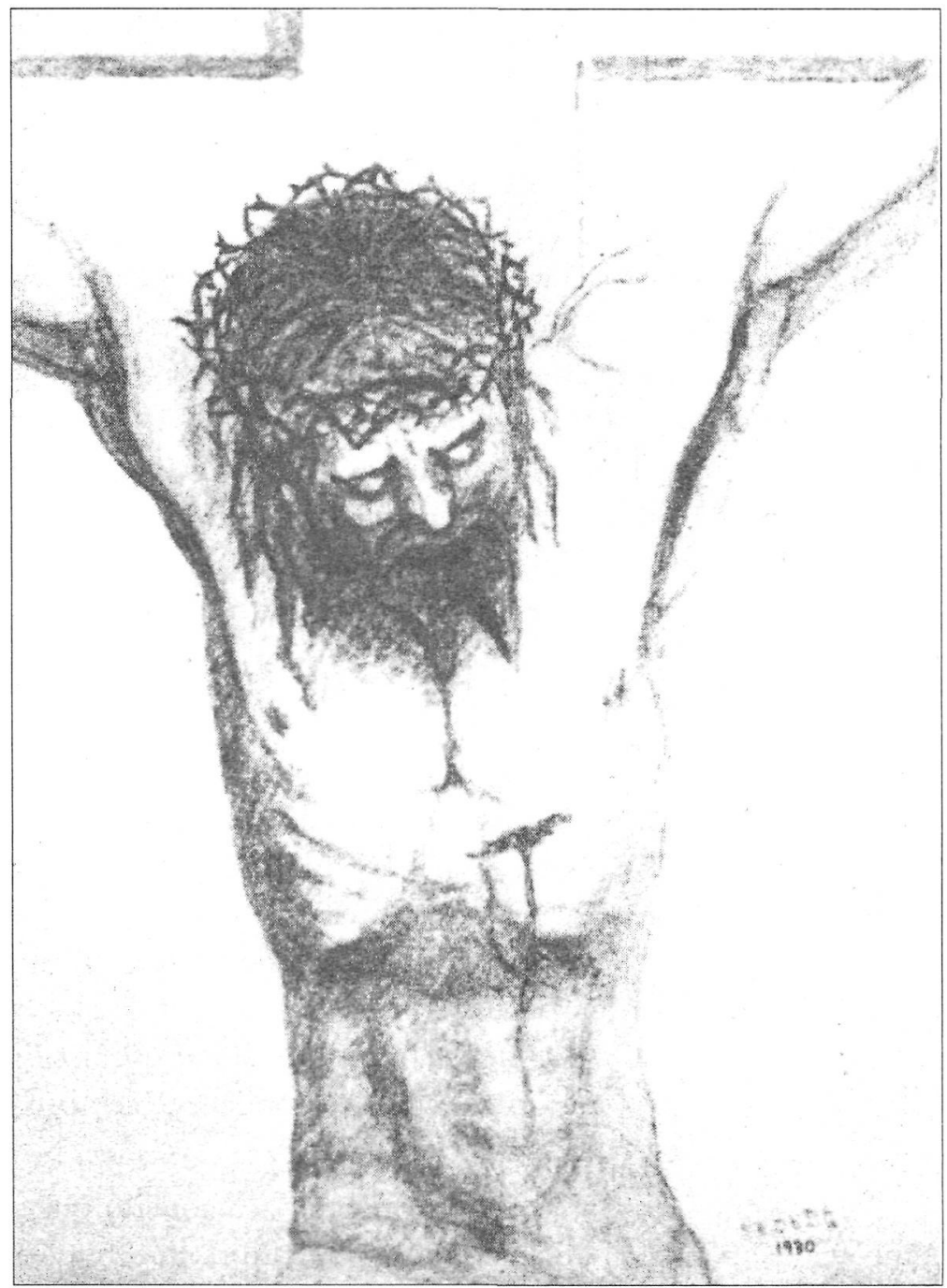

De Saint-Denys Garneau, Torse de Christ en croix. Fusain sur papier. 63,8 x 48,8 cm. Signé : de St.D.G. Daté : 1930. Collection G. H. Anciennement coll. Benoît Lacroix. "J'ai dessiné au fusain, durant les vacances de Noël, un torse de Christ en croix que j'ai fort bien réussi. Quand je dessine, quand je peins, je me sens quasiment du génie, je m’emporte et j'ai une satisfaction indéfinissable à voir se préciser peu à peu les images que je porte en moi. » 
présente ainsi : "J'avais peint le moulin mais je n'avais pas d'image de la vieille "chaufferie". J'en ai fait une aquarelle l'autre après-midi. Elle est assez bien réussie ${ }^{111}$. "

Enfin, deux fusains sur papier, tous deux datés : 1930. Le premier sur papier gris, un [Profil d'bomme à la barbe], signé : de St-D. Garneau ; chevelure longue, floue, suggérée, l'accent est mis sur le visage et l'œil, très vif. Très beau. Il fait partie de la série de fusains décrite plus haut. Enfin, un Torse de Christ en croix, signé : de St.D.G., que l'auteur présente ainsi : " J'ai dessiné au fusain, durant les vacances de Noël, un torse de Christ en croix que j'ai fort bien réussi. Quand je dessine, quand je peins, je me sens quasiment du génie, je m'emporte et j'ai une satisfaction indéfinissable à voir se préciser peu à peu les images que je porte en moi $^{112}$. »

\section{4) Le peintre parmi les peintres (biver 1932-printemps 1937)}

Une série de treize nus féminins d'après modèles vivants, des fusains surtout et quelques pastels, non datés et non signés, probablement réalisés à l'atelier de Edwin Holgate [février-mai 1932]. Ils font partie de la série de fusains décrite plus haut. Études du corps, dans des poses debout, assise et allongée, les visages sont inexistants ou à peine esquissés. Certains présentent de beaux volumes en rondeurs, bien proportionnés.

De Saint-Denys Garneau a préparé la maquette qui a remplacé la maquette originale de la couverture de la revue Vivre, fondée par Jean-Louis Gagnon et Philippe Vaillancourt (15 mai 1934-8 mars 1935). De Saint-Denys Garneau a fourni la maquette pour le troisième cahier de la première série, en octobre 1934, et une nouvelle version de la maquette, mieux réussie, à partir de novembre $1934^{113}$. 


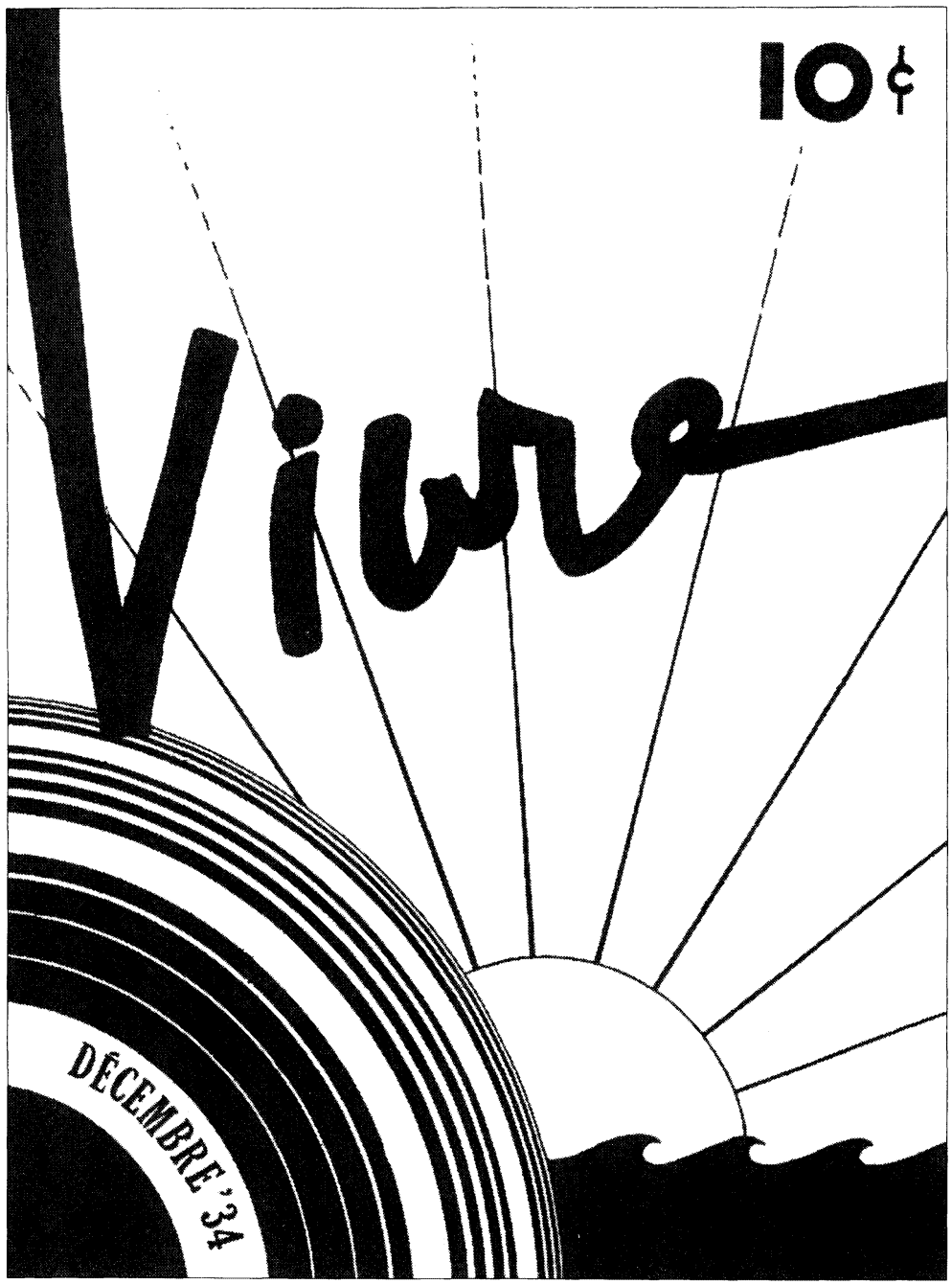

Couverture de la revue Vivre, d'après une maquette d'Hector de Saint-Denys Garneau. 23,7 x 17,3 cm. Décembre 1934. 
5) Le peintre en désenchantement (printemps 1937-1943)

Une autre manière apparaît, celle des textes illustrés pour le Livre I. Ou plutôt réapparaît puisque de Saint-Denys Garneau avait produit en 1928 son Recueil de poésies au contenu de poèmes et d'aquarelles. Cependant, dans cette dernière période, ce sont des œuvres humoristiques inspirées de faits vécus ${ }^{114}$.

Livre I : Le livre de bord Pingouin [ca 1938], 32 x $22 \mathrm{~cm}$, contient : Journal - Organe officieux de la navigation laurentienne. Illustré ; Jacques Cartier arrive à Québec (2 grandes illustrations); Paroles célèbres ; Poème «Les N Commandements du navigateur » (ill.) ; L'homme à bicyclette (ill.) ; L'homme aux cordages devant un mat (ill.) ; Esquisse de sirène (ill.) ; Cap aux diamants (ill.) ; Grand serpent de mer (ill.) ; Trois poissons dansant (ill.).

Livre II : Livre d'or offert par de Saint-Denys Garneau à sa tante Dorothy Fraser, épouse de Saint-Denys Prévost. [Été 1939]. 57 feuillets. Un seul utilisé. Sans texte. Composition représentant une partie du jardin de la maison Prévost à Sainte-Catherine, non loin du Manoir, après la transformation apportée par de Saint-Denys Garneau à la demande de sa tante.

Ces deux livres faisaient partie de l'exposition des manuscrits «Voici l'espace que j'habite » au Musée québécois de culture populaire, dans le cadre du Festival International de Poésie de Trois-Rivièrs, du 25 septembre au 12 octobre 2003.

\section{$* * *$}

Nous avons parlé du vœu de De Saint-Denys Garneau de contribuer à un renouveau, à une renaissance culturelle au Canada français. Il souhaitait être l'un de ceux qui élèveraient le seuil de la culture, de la littérature et de l'art parmi ses 
compatriotes, être l'un de ceux qui les conduiraient vers une vie culturelle et intellectuelle plus large, plus haute, plus universelle, en même temps que plus représentative de leurs désirs profonds réprimés.

Conscient que le travail de l'artiste évolue sur une très longue période de temps, que plusieurs doivent être sacrifiés sur l'autel avant que n'apparaisse le génie qui naitra éventuellement de leurs efforts, il n'imagine pas le succès spontané et immédiat et ne croit pas être ce génie auquel il rêvait de donner naissance. Il se situe parmi les forces obscures qui l'aideront à émerger. Et pourtant, sans qu'il ait jamais pu soupçonner qu'ainsi il en sera, de Saint-Denys Garneau peut prétendre à une place de choix dans la littérature québécoise. Alors que Gaston Miron le considère comme « le plus grand poète québécois ", que Gilles Marcotte va jusqu'à dire qu'il est peutêtre " notre plus grand écrivain ", il est maintenant reconnu comme le premier poète moderne du Québec.

En peinture, sa réputation ne va pas jusque-là. C'est à Borduas que revient le titre de premier peintre moderne du Québec. L'apport de De Saint-Denys Garneau, bien que considérable de par la volonté, la recherche, les labeurs et les forces qu'il a investis, malgré une santé fragile et souvent chancelante qui le laissait fréquemment pantelant après de longues séances de peinture, reste plus modeste, ce dont, d'ailleurs, lui, le critique d'art parfois génial, avait pleine conscience. Si de Saint-Denys Garneau n'a pas réussi, comme il le désirait, à être le premier à "renouveler l'art du paysage ", si le résultat de la somme de son travail n'a pas été celui qu'il espérait, cela ne lui enlève pas tout mérite. S'il n'est pas devenu le grand peintre qu'il rêvait d'être, il est infiniment plus qu'un peintre amateur. S'il n'a pas eu le temps d'acquérir un style bien personnel, sa peinture a toutefois évolué au fil des ans, à force de travail sur sa technique, de recherche dans les 
théories sur l'art. On perçoit une évolution dans ses peintures, qui part d'une reproduction plus fidèle, puis d'une composition plus libre jusqu'à une plus grande stylisation, un plus grand modernisme dans certaines. Cependant, cet artiste toujours en quête du devenir de soi, de la poursuite de soi dans l'au-delà de sa soif et de son désir, ne pourra jamais dans l'histoire de la peinture québécoise prétendre à la réputation d'un Borduas, d'un Lemieux ou encore d'un Pellan ou d'un Riopelle.

Tant de choses ont été dites après son choix du silence pour vivre désormais hors du diptyque littéraire et pictural. Qu'il est un poète en sursis, que son œuvre est inachevée, que sa vie même est inachevée, comme si toute œuvre et toute vie ne l'étaient pas. Il est mort jeune, à 31 ans. La somme d'une vie n'est pas que dans le nombre, ni même la qualité, de pages ou de peintures ou même d'ans. Si nous ne nous référons pas à ses critères, c'est ainsi forcément qu'il nous apparaîtra. Alain Grandbois, un autre poète, aussi peintre à ses heures, a mieux saisi le sens de son parcours :

On a dit, on dit encore que St-Denys Garneau n'a pas eu véritablement le temps de s'accomplir. Je ne le crois pas. Son art, si fragile soit-il, est l'expression même de son génie. L'heure du don n'est pas marquée par l'âge. [...] St-Denys Garneau nous a donné un jeune arbre de neige, de rêves et d'étoiles. Mais il a bouclé la boucle. C'est ainsi que les choses s'accomplissent. Dieu seul connaît la loi ${ }^{115}$.

De Saint-Denys Garneau n'a pas failli à sa vie, à sa mission. Sa vocation a évolué au prix de mille tourments, il s'est engagé dans la voie étroite, l'exigeante et impérieuse voie qui l'appelait, il s'est aventuré à la vivre, celle d'apparence si terrible qu'il en mourait parfois d'effroi. Malgré d'apparentes faiblesses, il est être de liberté à la volonté forcenée. Malgré 
des problèmes psychologiques certains, il refuse de les prendre en compte ou plutôt de les transformer en excuses pour expliquer ses difficultés, son mal de vivre, ou encore pour tenter de se disculper s'il lui arrive de tomber en chemin. Son aventure spirituelle et religieuse, il l'a vécue comme une longue marche, une longue montée sur une sente étroite et abrupte, une terre brûlée, peuplée de terribles découvertes, mais aussi d'étonnantes et grandioses révélations. Peu de joies mais d'intenses, la découverte de l'amour pur, de la charité, envers l'autre mais aussi envers lui-même : "J'ai trouvé mon âme [...] au fond de la charité. [...] J'ai vu comme je ne l'avais pas vue la fraternité des hommes [...] Je sais maintenant que j'existe, que rien, ni moi-même, ne peut me détruire ; car je ne suis à personne, ni à moi ; je suis à Dieu ${ }^{116}$. » Il en vient à l'acceptation de la souffrance, de la solitude, de la mort même. C'est parce qu'il était catholique et qu'il entrevoyait sa vie au sein du Corps Mystique que son aventure prend tout son sens. L'on n'est pas obligé de partager ni ses croyances ni sa démarche, mais son aventure ne peut être comprise que selon ses propres critères.

De Saint-Denys Garneau a vécu sa vie. C'était son lot sur terre et non celui d'autrui, de qui juge à l'aune de son regard, de son aventure et de l'arrangement de l'être selon un ordre qui n'existe pas pour de Saint-Denys Garneau. Il considérait de son devoir d'être ce qu'il a été, d'être à la frénétique poursuite de celui qu'enfin il est devenu à la limite du possible et même au-delà, juste à la frontière où l'Être l'a finalement pris en charge du $\operatorname{sien}^{117}$.

Un jour, il est parti en canot. Il a demandé si on pouvait l'accompagner. Il n'a trouvé personne pour le faire. Il s'en est allé à l'automne qu'il avait appris à aimer, figure de proue solitaire fonçant dans le courant haut et fort de la rivière Jacques-Cartier. Sans le savoir, seul, il partait au bout de lui- 
même, à la rencontre de la plénitude de son être et de l'Être qui l'allait rendre enfin possible. Son cœur malade depuis tant d'années a flanché. «Entre le ciel et l'eau ${ }^{118}$ », il est allé s'éteindre sur la grève rouge au cœur de "cette cathédrale d'arbres ${ }^{119}$ ", ces arbres qu'il avait longuement aimés et célébrés, les appelant par leur nom dans ses poèmes et dans son « Monologue sur l'arbre ${ }^{120} »$ et leur offrant mille formes et couleurs dans ses toiles si multiples à les représenter. Il a " poussé des racines » comme il l'avait rêvé dans la terre familière du pays qu'il aimait. Après son voyage terrestre avec " pour chapeau le firmament " et " pour monture la terre ${ }^{121}$ ", il commençait une autre vie et une autre œuvre, celle prise en charge par ses amis et autres amoureux de son art qui l'allaient répandre de par la terre et dans les cœurs.

\section{[Il nous est arrivé des aventures]}

Il nous est arrivé des aventures du bout du monde Quand on vient de loin ce n'est pas pour rester là (Quand on vient de loin nécessairement

$$
\text { c'est pour s'en aller) }
$$

Nos pieds n'étaient plus là pour nous attacher dans la terre

Ils nous attiraient tout le corps pour des journées

à perte de vue.

Il nous est arrivé des départs impérieux Depuis le premier jusqu'à n'en plus finir À perte de vue dans l'horizon renouvelé Qui n'est jamais que cet appel au loin

Ou cette barrière escarpée qui module le paysage

Qui fouette la rage de notre curiosité

Et ramasse en nous de son poids

Le ressort de notre bond 
On n'a pas eu envie de s'arrêter

On n'a pas eu trop de fatigues à dompter

Pour l'indépendance de nos gestes dans l'espace

Pour la liberté de nos yeux sur toute la place

Pour le libre bond de nos cœurs par-dessus les monts

Il en est qui n'ont pas voulu partir

Qui ont voulu ne pas partir, mais demeurer.

On les regarde on ne sait pas

Nous ne sommes pas de la même race.

Les autres, on est farouches, on est tout seuls

On n'a que l'idée dans la tête d'embrasser

On n'a que le goût de partir comme une faim

On n'est déjà plus où l'on est

On n'a rien à faire ici

On n'a rien à dire et l'on n'entend pas de voix

d'un compagnon ${ }^{122}$.

\section{NOTES}

${ }^{1}$ Cet article se voulait à l'origine un hommage à de Saint-Denys Garneau, à l'occasion du $60^{\mathrm{e}}$ anniversaire de sa mort, mais, pour des raisons indépendantes de notre volonté, il n'a pu paraitre en 2003. Nous le présentons donc en cette année du $10^{\mathrm{e}}$ anniversaire de la Fondation de Saint-Denys-Garneau. Comment serait-il possible de rendre justice, en un seul article, à tant de richesses garnéliennes sur l'art, théories et œuvres ? Ce ne l'est pas. Nous pouvons seulement donner des miettes, des pistes de recherche. Qu'un livre plus tard, si possible en 2004 ou en 2005, célébrera, espérons-le, avec plus de justesse. Il contiendra une étude, en plus détaillé, de ce qui se trouve ici, de même que des illustrations des peintures et dessins qui n'ont pas encore été reproduits (ce qui est donné en $\mathrm{II}^{\mathrm{e}}$ partie de cet article ne représente qu'une partie des inédits), une anthologie de textes sur l'art, une chronologie détaillée sur l'aventure artistique, une bibliographie, différents tableaux (la liste de ses lectures sur l'art, la liste des expositions de peintures, etc.) 
${ }^{2}$ Saint-Denys Garneau, Euvres, Texte établi, annoté et présenté par Jacques Brault et Benoit Lacroix, Montréal, Les Presses de l'Université de Montréal, 1971, p. 674 (désormais CEurres) ; Hector de Saint-Denys Garneau, Euvres en prose, Édition critique établie par Giselle Huot, Montréal, Fides, 1995, p. 676 (désormais Euvres en prose; ce dernier volume contient, en plus des textes déjà parus dans l'édition Brault-Lacroix, quarante-neuf textes de la période 19301938, dont quarante-six inédits); Hector de Saint-Denys Garneau, Poèmes et Proses (1925-1940), Avec des inédits (textes et illustrations), Choix et présentation de Giselle Huot, Montréal, Éditions de l'Outarde, 2001, p. 214 (désormais Poèmes et Proses).

${ }^{3}$ Les deux albums qui rassemblent une partie des œuvres picturales de l'écrivain-peintre : Saint-Denys Garneau, Regards et Jeux dans l'Espace, Conception et réalisation de Henri Rivard, Présentation d'Anne Hébert, comportant le texte de l'édition originale [1937] avec la reproduction de 35 tableaux, Montréal, Fides, 1993, 208 p. ; L'Univers de Saint-Denys Garneau, le peintre, le critique, Présentation de France Gascon, Montréal / Joliette, Boréal / Musée d'art de Joliette, 2001, 111 p. Reproduction en couleurs de 50 œuvres de De SaintDenys Garneau et de 7 œuvres de peintres sur lesquels il a écrit. Sur la critique d'art et la production picturale, voir : Georges Cartier, « Catalogue des peintures, aquarelles, pastels et fusains de Saint-Denys Garneau», Annexe dans BioBibliographie de Saint-Denys Garneau, Préface de Robert Élie, Montréal, École de bibliothécaires, 1952, pp. $70-74$ (premier inventaire partiel de 30 œuvres); Georges Cartier, «Le tombeau d'un poète. Paysage de Saint-Denys Garneau ", L'Action universitaire, vol. 20, $\mathrm{n}^{\circ} 1$ (octobre 1953), pp. 26-34 ; Georges Cartier, « Paysage de Saint-Denys Garneau », Lectures, vol. 10, $\mathrm{n}^{\circ} 5$ (janvier 1954), pp. 196-201; Jean-Pierre Duquette, "Saint-Denys Garneau dessinateur et peintre », Littératures, $\mathrm{n}^{\circ}$ 12, "Saint-Denys Garneau parmi nous », 1994, pp. 45-51 ; François Hébert, "Paysage sans cadre », Liberté, vol. 37, n 1, 223 (février 1996), pp. 81-90 ; François Hébert, « Le peintre Saint-Denys Garneau ", Liberté, vol. 40, n 4, 238, août 1998, pp. 4-24 (huile d'Hector de SaintDenys Garneau sur la page couverture) ; France Gascon, «Une autre façon d'être peintre ", dans L'Univers de Saint-Denys Garneau, le peintre, le critique, pp. 12-25 ; Hector de Saint-Denys Garneau, Recueil de poésies. Inédit de 1928, Présentation de Giselle Huot, Québec / Montréal, Éditions Nota bene / Éditions de l'Outarde, 2002, 188 p. (10 poèmes illustrés de 7 aquarelles et deux séries de "Pensées ») ; Serge Proulx, "La "Poésie" de Saint-Denys Garneau et la crise identitaire au Canada français ", Liberté, vol. 45, $\mathrm{n}^{\circ}$ 1, 259 (février 2003), pp. 89-102; Catherine Desgagnés, Déséquilibres et harmonie : le discours sur l'art de Saint-Denys Garneau, mémoire de maîtrise, Université de Montréal [à venir] ; Andrée-Anne Giguère, Saint-Denys Garneau essayiste. Étude 
des textes en prose publiés entre 1927 et 1938, mémoire de maitrise, Université Laval [à venir].

${ }^{4}$ Ses prénoms sont «Hector » et « de Saint-Denys » et non «Saint-Denys »; voir à ce sujet Euvres en prose, pp. xv-xix ou encore Poèmes et Proses, p. 368.

${ }^{5}$ Poème «Le jeu », Euvres, p. 10 ; Poèmes et Proses, p. 33.

${ }^{6}$ Hermine Prévost-Garneau, «Souvenirs par mémoire involontaire », dans Mémorial, Inédits de De Saint-Denys Garneau, de parents et d'amis, Conçu et réalisé par Giselle Huot et Benoît Lacroix, Cahiers de Saint-Denys Garneau, ${ }^{\circ} 1$, Saint-Hippolyte / Montréal, Éditions du Noroît / Fondation de Saint-Denys-Garneau, 1996, p. 21 (désormais Mémorial).

${ }^{7}$ Euvres en prose, p. 193.

${ }^{8}$ Voir infra, «II La production artistique ». Très tôt, encouragé dans ses efforts artistiques et littéraires, c'est à Sainte-Catherine également qu'il compose le premier poème que nous lui connaissons, "Le pavyon de la France », peutêtre aussi de 1920. C'est là encore qu'il connait ses premiers succès de scène vers 1923 ou peut-être avant et aussi plus tard, dans les années 1930-1936. C'est là également qu'il fera l'apprentissage de la photographie vers 1940.

${ }^{9}$ L'ancêtre de la première génération au Canada est Auguste-Franz Globensky, fils de Joseph Globensky, secrétaire du roi de Prusse. L'arrière-grand-père de l'auteur, Léon Globensky (1807-1879), percepteur de douanes à Montréal, est le fils d'Auguste-Franz, le frère du lieutenant-colonel Maximilien Globensky et l'oncle de Charles-Auguste-Maximilien, l'auteur de La Rébellion de 1837 à Saint-Eustache.

${ }^{10}$ On peut admirer cette toile, malheureusement reproduite en noir et blanc, représentant Élodie Globensky, aux côtés du portrait de son amie Azélie Papineau, dans Raymond Vézina, Napoléon Bourassa (1827-1916). Introduction à l'étude de son art, Montréal, Éditions Élysée, 1976, p. 61.

${ }^{11}$ Conférence non retrouvée sur Alfred Garneau et Louis Fréchette prononcée à la Société canadienne de science et d'art, dont l'organe est La Revue scientifique et artistique (voir Euvres en prose, p. 159).

${ }^{12}$ Dans ses «Notes pour mes mémoires », il insère la date légèrement erronée de la mort de sa grand-mère (avril 1927) parmi les événements importants qu'il voulait y relater (CEuvres en prose, pp. 159, 261-262).

${ }^{13}$ « Napoléon Bourassa doit se réjouir, lui, le précurseur de l'enseignement public de l'art chez nous, de voir enfin sur pied cette école à laquelle il rêvait dès avant 1880 , et dont il fit tout pour hâter la création, au moyen de voyages 
d'étude, d'articles et de rapports. » Olivier Maurault, Marges d'bistoire, Montréal, Librairie d'action canadienne française, 1929, t. I, L'Art au Canada, pp. 78.

${ }^{14}$ Le directeur-fondateur est le Français Emmanuel Fougerat, artiste-peintre, auparavant directeur-fondateur de l'École régionale des beaux-arts de Nantes. Charles Maillard lui succédera en 1925. Voir Fernand Harvey, «La politique culturelle d'Athanase David, 1919-1936», Les Cabiers des Dix, $\mathrm{n}^{\circ} 57$ (2003), pp. 56-64.

${ }^{15}$ Plusieurs écrivains d'ailleurs et d'ici font aussi du dessin ou de la peinture. Pour n'en nommer que quelques-uns du Québec : Jacques Brault, Louis Caron, Cécile Chabot, Pierre Gauvreau, Roland Giguère, Charles Gill, Alain Grandbois et Sergio Kokis. En 1996-1997, se tenait au Domaine Cataraqui (Sillery, Québec) l'exposition "Art \& Lettres », réunissant des toiles de De Saint-Denys Garneau, de Pierre Gauvreau et de Roch Plante (pseudonyme de Réjean Ducharme). Voir Roland Bourneuf, «La double pratique.[...] SaintDenys Garneau et Giguère », Littérature et Peinture, Québec, L'Instant même, 1998, pp. 128-131.

${ }^{16}$ «La proclamation des lauréats de l'École des Beaux-Arts », Le Canada, 26 mai 1925 , p. 8 .

${ }^{17}$ Voir Roger Blais, Jean Palardy, peintre témoin de son époque, Montréal, Stanké, 1993, p. 24 ; François-Marc Gagnon, Paul-Émile Borduas, Montréal, Musée des beaux-arts de Montréal, 1988, p. 33 ; Edward P. Lawson, «Jean-Paul Lemieux raconte sa jeunesse », La Presse, 15 septembre 1967, p. 12.

${ }^{18}$ Voir « Notes pour mes mémoires », 7 mai [19]28, CEuvres en prose, pp. 157159.

${ }^{19}$ "Peintres français à la Galerie Scott», La Relève, $3^{\mathrm{e}}$ série, $2^{\mathrm{e}}$ cahier, décembre 1936, pp. 45-50 ; Euvres, pp. 283-284 ; CEures en prose, pp. 101-102. Le nom du professeur n'est pas mentionné dans La Relève, mais la version manuscrite du cahier VI du Journal l'identifie clairement : « M. St-Charles professeur à l'école des Beaux-Arts, n'a eu de joie d'aucune sorte [...]» (CEuvres en prose, $\mathrm{p}$. 1000).

${ }^{20}$ Cuvres en prose, pp. 374-375.

${ }^{21}$ Ibid., pp. 375-376.

${ }^{22}$ Après avoir commencé son cours classique au collège Sainte-Marie en septembre 1923, de Saint-Denys Garneau ne peut y retourner à cause de problèmes de santé. Il passe les deux années suivantes en anglais au collège Loyola 
(1924-1926), toujours chez les jésuites. En 1926-1927, il suit des cours privés afin de compléter ses Éléments latins et faire sa Syntaxe. Par la suite, la fréquentation des collèges Sainte-Marie $(1927,1929,1930-1933)$ et Jean-deBrébeuf $(1928,1929-1930)$ sera aussi entrecoupée de cours avec des professeurs privés à cause de sa santé.

${ }^{23}$ Il ajoute que c'est "par Dieu qu'on atteint le Beau», puisque «la poésie tend à tout ramener aux sources premières, et que La Source des sources est le Seul Dieu ». (Euvres en prose, p. 198)

${ }^{24}$ Journal, Euvres en prose, p. 404.

${ }^{25}$ Octobre 1929, Euvres en prose, p. 193 ; Poìmes et Proses, p. 112.

${ }^{26}$ "L'exposition du printemps à la Galerie des Beaux-Arts ", La Revue scientifique et artistique, $\mathrm{n}^{\circ} 25$ (avril 1930), pp. 7-11, dans CEuves en prose, pp. 15-24.

${ }^{27}$ À Françoise Charest, 31 mars [1930], CEuvres, pp. 825, 827-828.

${ }^{28}$ À Françoise Charest, 17 avril 1930, CEuvres, p. 832 ; Poèmes et Proses, p. 117. ${ }^{29}$ CEuvres, p. 828.

${ }^{30}$ À Françoise Charest, 25 avril 1930, Euvres, pp. 837-838.

${ }^{31}$ «Et que je sois une œuvre d'art. » (28 septembre 1932, CEuvres en prose, p. 914).

32 À Charles Saint-Arnaud, s.j., Euvres, p. 1003 ; Poèmes et Proses, pp. 114-115.

${ }^{33}$ Le Cercle de Science et d'Art dont l'organe est La Revue scientifique et artistique, le Cercle de la Pie créé par Pierre Dansereau, le Cercle Crémazie créé par André Laurendeau, puis le groupe de La Relève (1934) avec Robert Charbonneau, Paul Beaulieu, Claude Hurtubise, auxquels se joignent André Laurendeau, Robert Élie, Jean Le Moyne et d'autres (voir Euvres en prose, pp. xxxvi-xxxvii, xl-xlii, 9-10, 159, 160-161).

${ }^{34}$ À Charles Saint-Arnaud, s.j., CEuvres, pp. 1003-1004 ; Poèmes et Proses, p. 115.

${ }^{35}$ Lettre du 11 janvier 1932 à Jacques Cousineau, s.j., dans Giselle Huot, «Des femmes, des professeurs et des amis. Poème et lettres inédits de Saint-Denys Garneau ", Les Cabiers d'histoire du Québec au XX siècle, $\mathrm{n}^{\circ} 1$ (hiver 1994), pp. 55-56.

${ }^{36}$ Ibid., p. 56. 
37 À Jean Le Moyne, [27 et 28] janvier 1934, dans Saint-Denys-Garneau, Lettres à ses amis, Avertissement de Robert Élie, Claude Hurtubise et Jean Le Moyne, Montréal, Éditions HMH, 1967, p. 96 (désormais Lettres).

${ }^{38}$ Ibid., pp. 98-99.

${ }^{39}$ Quelques exemples de livres qu'il lit : Le Traité du paysage de Léonard de Vinci ; Introduction à la peinture bollandaise de Paul Claudel; Histoire de la peinture française an XIX' siècle (1793-1890) de Louis Dimier ; Histoire de l'art d'Élie Faure; Raphaël de Louis Gillet; Le Tintoret sous la direction de Henry Roujon. (Sur ces lectures, voir Benoît Lacroix, «Sa bibliothèque privée», Études françaises, vol. 20, n 3 (1984), «Relire Saint-Denys Garneau », hiver 1984-1985, pp. 97-111.) Il possède plusieurs numéros de la revue Formes. Il lira et relira Art et Métaphysique de Jacques Maritain. Voir aussi Roland Bourneuf, Saint-Denys Garnean et ses lectures européennes, Québec, Les Presses de l'Université Laval, 1969, passim (voir index).

${ }^{40}$ Voir Roger Blais, Jean Palardy, p. 23.

${ }^{41}$ L'on sait peu de choses sur cet atelier, si ce n'est par les quelques détails qu'il a laissés dans deux lettres, dont la dernière nous apprend également d'où proviennent les photos du De Saint-Denys Garneau au béret (Lettres, pp. 41 et 42). Voir aussi Jacques Roy, L'Autre Saint-Denys Garneau. Suivi de cinq lettres inédites de Saint-Denys Garneau, Québec, Éditions du Loup de Gouttière, [1993], p. 56 et les photos pp. $46-48$.

${ }^{42}$ Voir Lettres, pp. 79 ss. Jori Smith a publié un livre sur leur séjour en Charlevoix : Charlevoix County, 1930, Penumbra Press, 1998, 110 p., ill.

${ }^{43}$ Originaire de l'Ontario, Holgate avait été l'un des élèves de William Brymner à l'Art Association de Montréal, dont les meilleurs allaient étudier en Europe, où il fréquentera plusieurs académies de Paris. Pendant deux ans, il fera partie du Groupe des Sept dont il était le seul membre résidant au Québec, où il s'installe définitivement après ses voyages d'Europe. Il introduit la figure humaine et le nu dans la peinture canadienne.

${ }^{44}$ Euvres en prose, p. 376.

${ }^{45}$ Entrevue avec Edward P. Lawson, «Jean-Paul Lemieux raconte sa jeunesse », La Presse, 15 septembre 1967, p. 12

${ }^{46} \mathrm{Ibid}$. Sur l'art du nu, voir Jacques de Roussan, Le NU dans l'art au Québec, LaPrairie (Québec), Éditions Marcel Broquet, 1982, 223 p., ill.

${ }^{47}$ À Jean Le Moyne, Lettres, p. 39. 
${ }^{48}$ Marie Carani, «L'École des beaux-arts de Montréal. Edwwin Holgate et les amitiés durables de l'École des beaux-arts de Montréal », Jean-Paul Lemieux, Québec, Musée du Québec / Les Publications du Québec, 1992, p. 51.

49 "Jean-Paul Lemieux raconte sa jeunesse », p. 12.

${ }^{50}$ « Parmi les étudiants que l'artiste accueille à son studio au cours de ses six années d'enseignement à l'École des beaux-arts, citons, outre Lemieux, Jori Smith, Stanley Cosgrove, Paul-Émile Borduas, l'écrivain Saint-Denys Garneau et le $\mathrm{D}^{\mathrm{r}}$ Norman Bethune. » (Dennis Reid, Edwin Holgate, Ottawa, Galerie nationale du Canada,1976, p. 13.)

${ }^{51}$ Robert Élie, Borduas, Montréal, Éditions de l'Arbre, 1943, 24 p., ill.

${ }^{52}$ Muhlstock ne fait pas partie de ce $56^{\mathrm{e}}$ Salon, mais ses dessins étaient exposés dans la «Print Room » de la Galerie des Arts (l'actuel Musée des beaux-arts), du 16 au 28 novembre 1935.

${ }^{53}$ Eurres en prose, p. 93.

${ }^{54}$ Sur Louis Muhlstock, on peut consulter, entre autres Jennifer Couëlle, $U n$ dessinateur Louis Mublstock The Draughtsman, Montréal, Saidye Bronfman Centre, 96 p. ; Pierre Murgia, Louis Gauvin et Nancy Trépanier, Mublstock, Québec, Musée du Québec, 1995, 48 p. ; Serge Wagner, Louis Mublstock, OC, LLD, CGP, «Un peintre dans le siècle », Hull, Galerie Montcalm, 1995, 31 p.

${ }_{55}$ "Chronique des beaux-arts [II] : Louis Muhlstock », La Relève, $2^{\mathrm{e}}$ série, $5^{\mathrm{e}}$ cahier, janvier 1936, pp. 158-160; Euvres en prose, pp. 96-100.

${ }^{56}$ «[...] Au petit salon de l'Art Association, où vous exposiez vos dessins, ce fut, pour Monsieur de Saint-Denys Garneau et moi la satisfaction d'un long désir. Nous sommes jeunes et pourtant de toutes les expositions que nous avons pu voir combien peu de noms à retenir. Devant vos dessins, il ne s'agissait plus de retenir un nom, mais d'aimer une œuvre vivante. M. Garneau doit publier dans le prochain numéro de La Relève (organe d'un groupe dont nous faisons partie) un article qui vous dira toute notre admiration [...] 》 (Lettre conservée précieusement par Louis Muhlstock et reproduite dans le Mémorial, p. 83.) Robert Élie raconte cette première rencontre, Ibid., pp. 84-85.

${ }^{57}$ Ibid., pp. 80-81.

${ }^{58}$ Ibid., p. 81.

${ }^{59}$ Ibid. 
${ }^{60}$ À Gertrude Hodge, [29 ?] juillet 1936. Lettre inédite à paraître dans Hector de Saint-Denys Garneau, Correspondance, Édition critique par Giselle Huot.

${ }^{61}$ Sur diapositive prise par Georges Cartier (voir supra, n. 3).

${ }^{62}$ Le Prix Louis-Muhlstock a été décerné par l'intermédiaire de la Fondation de Saint-Denys-Garneau, en collaboration avec le Centre d'études québécoises de l'Université de Montréal, à un étudiant auteur d'un texte inédit sur de Saint-Denys Garneau, de 1997 jusqu’à sa mort en 2001.

${ }^{63}$ Parmi les associations les plus importantes qu'a créées John Lyman, le « Groupe de l'Est» au printemps de 1938, et, en 1939, la Contemporary Art Society (la Société d'art contemporain), dont il est président et Borduas, viceprésident ; Robert Élie en sera membre aussi.

${ }^{64}$ Louise Dompierre, John Lyman, 1886-1967, Kingston, Agnes Etherington Art Centre / Queen University, 1986, p. 62.

${ }^{65}$ Paul Dumas, Lyman, Montréal, L'Arbre, 1944, p. 12.

${ }^{66}$ John Lyman, Morrice, Montréal, L'Arbre, 1945.

${ }^{67}$ Je vispar les yeux, 22 août 1958, 30 minutes, produit par Fernand Dansereau et réalisé par Léonard Forest; télédiffusé la première fois le 26 novembre 1958 dans la série Profils et paysages, sous le titre de John Lyman, peintre.

${ }^{68}$ Journal, [avril-début mai 1937], Euvres en prose, p. 499.

${ }^{69}$ Pour cette citation et la réponse des autres peintres qu'il rapporte, voir Journal, Euvres en prose, pp. 622-623.

${ }^{70}$ Dompierre, John Lyman, p. 52. « Méconnu, mais personnage important de la peinture québécoise John Lyman le fut surtout par sa culture, sa connaissance des milieux et des mouvements artistiques européens. En fait, il fut le personnage-clé d'un mouvement qui attira les peintres hors de l'influence de la peinture patriotique du groupe des Sept, peinture qui, selon Lyman, menaçait de devenir une "peinture-souvenir". » (Hedwidge Asselin, Inédits de John Lyman, Montréal, Ministère des affaires culturelles / Bibliothèque nationale du Québec, 1980, pp. 19, 20 et 15.)

${ }^{71}$ «Moi - Art - Peinture », Journal, [Fin septembre 1936], CEuvres en prose, p. 453.

${ }^{72}$ «Surréalisme - Art - Esthétique», Journal, Euvres en prose, pp. 403-406.

${ }^{73}$ " Expérience », [Fin septembre-1 ${ }^{\mathrm{er}}$ octobre 1936], Journal, CEuvres en prose, pp. 458-459. 
74 À André Laurendeau, 30 août 1933, CEuvres, pp. 930-931; Lettres, p. 86.

${ }^{75}$ CEuvres en prose, pp. 591-599 ; extraits dans Poèmes et Proses, pp. 256-260.

${ }^{76}$ Voir supra, n. 36.

77 À Jean Le Moyne, 1937, Lettres, p. 249.

${ }^{78}$ À Robert Élie, [début] septembre 1936, Lettres, p. 225 ; Poèmes et Proses, p. 210. Voir aussi CEuvres, p. 942 ; Poimes et Proses, p. 204.

${ }^{79}$ Voir « Rencontres, paysans », CEurres en prose, p. 461 ; Poèmes et Proses, p. 219.

${ }^{80}$ À Jean Le Moyne, 20 mars 1934, Lettres, p. 125.

${ }^{81}$ Extrait d'une lettre à André Laurendeau, dans Giselle Huot, « Des femmes, des professeurs et des amis... ", p. 61 ; Poìmes et Proses, p. 234.

${ }^{82}$ À ce sujet, voir Euvres en prose, p. xvii, n. 8.

${ }^{83}$ Elle est mentionnée dans la catégorie des peintures à l'huile, aquarelles et pastels : «Garneau, St. Denys, 353 Olivier Ave, Westmount, P.Q. / 118 - Ciel en Automne... N[ot] F[or] S[ale] ». (Catalogue of the Fifty-Fourth Spring Exbibition. March 18th, to April 11th, inclusive, [Montreal], Art Association of Montreal, 1937, p. 8.) De Saint-Denys Garneau se retrouvait là en compagnie de beaucoup de peintres mentionnés dans ses chroniques de beaux-arts : Lilias Torrance-Newton, qui présente un portait de Louis Muhlstock, Alphonse Jongers, Adam Sherriff Scott, Alberta Cleland, Agnès Lefort, Frank Iacurto, Harold Beament, Berthe Des Clayes, Paul Caron, A. Bieler, Coburn, Frank-S. Panabaker et Robert Pilot.

${ }^{84}$ Valdombre [pseudonyme de Claude-Henri Grignon], « Regards et jeux dans l'espace », En Avant !, 26 mars 1937, p. 3. Voir Euvres en prose, pp. 126-127 et 497-498.

${ }^{85}$ CEuvres en prose, p. 639 ; Poèmes et Proses, p. 276.

${ }^{86}$ Extrait d'une lettre à Robert Élie, Lettres, p. 225 ; Poèmes et Proses, p. 210.

${ }^{87}$ Sur ce voyage, voir CEuvres en prose, pp. 512-514, n. 112.

${ }^{88}$ France, juillet 1937. Notes écrites sur le carton déplié d'un paquet de cigarettes Export. CEurres en prose, pp. 927-928 ; Poèmes et Proses, pp. $241-242$.

${ }^{89}$ Journal, septembre 1937, CEurres en prose, p. 528.

90 À Jean Le Moyne, 5 octobre 1937, Lettres, p. 312.

${ }^{91}$ Journal, [18] mai 1935, CEuvres en prose, p. 274. 
${ }^{92}$ CEuvres en prose, p. 622.

${ }^{93}$ CEuvres en prose, p. 274.

94 À Jean Le Moyne, 1937, Lettres, pp. 248-249.

95 Ibid., p. 252.

${ }^{96}$ Extrait d'une lettre non envoyée de Robert Élie à de Saint-Denys Garneau, [ca 1937 ss], publiée dans Mémorial, pp. 90-91.

${ }^{97}$ Antoine Prévost, “ "On va voir de Saint-Denys”. Souvenirs d'un jeune cousin ", Euvres en prose, pp. 984-985. Il a aussi écrit un essai De Saint-Denys Garneau, l'enfant piégé. Récit biographique, Montréal, Boréal, 1994, 239 p. Pour le cinquantième anniversaire du décès de son cousin, en 1993, Antoine Prévost s'était inspiré de Regards et Jeux dans l'Espace pour créer une série de toiles magnifiques. Exposées d'abord dans le cadre du Festival International de Poésie de Trois-Rivières à l'automne de 1993, elles le seront ensuite à Toronto pour le Colloque « Saint-Denys Garneau, cinquante ans après » (19-21 novembre 1993) et, enfin, au Musée Marc-Aurèle Fortin à Montréal (2 novembre-11 décembre 1994), le lancement de son livre De Saint-Denys Garneau, l'enfant piégé était jumelé au vernissage le 2 novembre. Voir Paul Gladu, « Antoine Prévost. Gros plan sur Saint-Denys Garneau », Magazin'Art, vol. 7, n 2 (hiver 1994-1995), pp. 48-51, ill.

${ }^{98}$ CEures, p. 941 ; Poèmes et Proses, p. 203.

${ }^{99}$ Robert Élie, op. cit., p. 758.

${ }^{100}$ Félix-Antoine Savard s'est longtemps senti coupable de ne pas l'avoir fait, jusque dans son Journal et Souvenirs, Montréal, Fides, 1973, t. I, p. 78.

${ }^{101}$ Robert Élie, «Témoignage sur Borduas », Liberté, vol. 4, n ${ }^{\text {os }} 19-20$ (janvierfévrier 1962), p. 17 ; reproduit dans Robert Élie, Euvres, pp. 604-605. Lorsque son livre sur Borduas paraît,en 1943, Robert Élie en envoie un exemplaire à Hermine et Paul Garneau, les parents de De Saint-Denys Garneau. Hermine Prévost Garneau lui répond le 16 février 1944 : «J'ai lu avec beaucoup d'intérêt votre plaquette sur le peintre canadien Borduas. » Elle termine sa lettre en souhaitant aller admirer ses toiles avec son mari, non sans avoir avoué auparavant : «je n'ai pu me défendre de faire un rapprochement entre votre peintre et de Saint-Denys. Tous deux ne chantent-ils pas "les splendeurs de la lumière". Ils s'expriment librement dans un espace qu'aucune règle ne vient limiter [...]Vous pénétrez l'âme de votre peintre comme vous avez pénétré celle de "votre meilleur ami". " 
${ }^{102}$ Extrait de " Au delà du refus ", Revue dominicaine, vol. 55, t. 2 (juillet-août 1949), pp. 5-18 et septembre 1949, pp. 67-78, dans Robert Élie, CEuvres, p. 589.

${ }^{103}$ Gilles Marcotte, «Pauvreté d'Hector de Saint-Denys Garneau (1912-1943) », dans Gilles Routhier et Jean-Philippe Warren, dir., Les Visages de la foi. Figures marquantes du catholicisme québécois, Montréal, Fides, 2003, p. 115.

${ }^{104}$ Jean-Pierre Duquette en a fait une description sommaire dans "SaintDenys Garneau dessinateur et peintre », Littératures, Montréal, $\mathrm{n}^{\circ} 12$, «SaintDenys Garneau parmi nous », 1994, pp. 45-51.

${ }^{105}$ Lever de lune. Aquarelle. 13 x 19 cm. Non signé. Daté : 6 fév. 1920. En tête, ajout postérieur : Ma première peinture, voir note au verso. Au verso, nous y lisons : « À ma chère maman un cado de son petit / fice de St-Denys, garneau. / 6 fév. 1920 (7 ans) ». Neuf ans après, le 4 octobre 1929, dans Mon Journal. Mon bistoire, il raconte les circonstances de cette création (CEurres en prose, pp. 193194 ; Poìmes et Proses, pp. 112-113).

${ }^{106}$ Pour la description, voir CEuvres en prose, p. 900.

${ }^{107}$ Il est spécifié : «Les travaux non primés pourront être rendus aux élèves après l'exposition de fin d'année. " (École des beaux-arts de Montréal. Palmarès, Province de Québec, Quatrième année, 28 mai 1927, p. 30. Archives UQAM, Fonds École des beaux-arts de Montréal, 5P5/2.)

${ }^{108}$ On peut l'admirer dans le Paul-Émile Borduas de François-Marc Gagnon, 1988, p. 67, qui note : «il s'agit du moulage d'une anse du fameux Vase de la Paix exécuté à Versailles en 1684 par Jean-Baptiste Tubi dit le Romain ».

${ }^{109}$ Reproduites dans Poèmes et Proses, p. 101.

${ }^{110}$ Signé : de St.D.G. Daté : 1928. Collection BAC, provenant de la Collection Antoine Prévost, Montréal. Il faisait partie de l'exposition des manuscrits "Voici l'espace que j'habite», tenue au Musée québécois de culture populaire, dans le cadre du Festival International de Poésie de Trois-Rivières, du 25 septembre au 12 octobre 2003.

${ }^{111}$ Lettres, p. 9.

${ }^{112}$ Lettre du 14 janvier 1930 au père Charles Saint-Arnaud, s.j., CEuvres, p. 1003 ; Poèmes et Proses, pp. 114-115. Ce Christ en croix, que Madame Garneau avait accroché au Manoir et devant lequel elle faisait prier ses petits enfants, peut évidemment être inspiré de n'importe quel crucifix ou de peintures. Toutefois, il a peut-être été inspiré par la grande croix du cimetière paroissial à Sainte-Catherine, qui tient lieu de monument funéraire à plusieurs membres 
de sa famille et à lui-même ; sa mère y fait apposer un Christ de bronze en 1928. (CEurres en prose, p. 168, n. 80 ; Poèmes et Proses, p. 104 (photo).) Il y a un poème «Cimetière », daté du 5 juin 1929, dans lequel on lit : «Un grand Christ qui saigne, / Sur une grande croix / De bois, / Comme saigne / Le grand soleil couchant / Qui saigne / Comme saigne / Le grand Christ mourant!» (CEurres, p. 77). Mme Garneau avait fait préparer par Georges Beullac une photo où l'on voit d'une part ce fusain du Christ en croix et, d'autre part, le poème « [Et je prierai ta grâce] » (CEuvres, p. 188 ; Poèmes et Proses, p. 85.)

- [Paysage champêtre]. Aquarelle. 9 x $11 \mathrm{~cm}$. Signé : de St.D.G. Non daté : ca 20 avril-5 mai 1930. Collection Giselle Huot, Montréal. Reproduction dans Études françaises, hiver 1984-1985, p. 98. Sur carte avec envoi de vœux de Pâques à Françoise Charest (CEuvres, p. 834).

- Aquarelle. 14,2 cm x 11,5 cm. Non signé. Non daté [1930]. Collection Giselle Huot, Montréal. Reproduction en couleurs sur la couverture de Poèmes et Proses.

${ }^{113}$ Voir Jean-Louis Gagnon, Les Apostasies, Montréal, La Presse, 1985, t. I, Les Cogs de village, pp. 68-69, qui raconte comment de Saint-Denys Garneau en est venu à la concevoir. Sur Vivre et les Cabiers noirs, voir André Beaulieu et Jean Hamelin, La Presse québécoise des origines à nos jours, Québec, Les Presses de l'Université Laval, 1984, t. VI (1920-1934), p. 293 et t. VII (1935-1944), p. 29. La revue sera remplacée par les Cabiers noirs ( 9 septembre 1935, probablement le seul numéro), publiés aux Éditions de Vivre, la reproduction de ce Vivre étant celui préparé par de Saint-Denys Garneau pour la revue de ce nom.

${ }^{114}$ Collection BAC, provenant de la Collection Denys Prévost, fils, pour le premier livre et de la Collection Antoine Prévost, pour le deuxième. Ces deux livres faisaient partie de l'exposition des manuscrits « Voici l'espace que j'habite » au Musée québécois de culture populaire, dans le cadre du Festival International de Poésie de Trois-Rivièrs, du 25 septembre au 12 octobre 2003. Le Pingouin est le yacht de son oncle Saint-Denys Prévost, acquis vers 1932, et sur lequel de Saint-Denys Garneau a fait plusieurs voyages au plus ou moins long cours. Voir CEurres en prose, pp. 692-695, pour les textes qu'il contient. Le second livre contient une composition représentant une partie du jardin de la maison Prévost à Sainte-Catherine, non loin du Manoir, après la transformation apportée par de Saint-Denys Garneau à la demande de sa tante. Il explique cette modification apportée au paysage dans Lettres, p. 406, 1[3] juillet 1939. 
${ }^{115}$ Alain Grandbois, «Saint-Denys Garneau », Notre temps, 17 mai 1947, p. 3, repris dans Alain Grandbois, Proses diverses, Édition critique par Jean Cléo Godin, Les Presses de l’Université de Montréal, 1996, p. 377.

${ }^{116}$ «Et alors, parce que j'ai pu accepter l'autre, le terne, le morne, le sec, celui qui n'existait pas auparavant dans mon appréciation de moi-même, parce que j'ai accepté le mort, je puis accepter le vivant; parce que tous les deux, le poète et l'autre, participent de mon âme. Et c'est comme une acceptation du monde [...] 》 (Lettres, pp. 224 et 225 ; Poèmes et Proses, pp. 208 et 209.)

${ }^{117}$ «Saint-Denys Garneau, comme quelques-uns de ses amis, mais avec une avidité toute particulière, faisait, par rapport au milieu culturel du Canada français, une expérience de solitude qui ne pouvait être qu'extrêmement cruelle. Celui qu'il voulait être, l'écrivain et le spirituel à la fois, était dans la culture de son pays un être entièrement nouveau, porteur de questions auxquelles rien, dans sa propre tradition, ne pouvait faire écho. » Marcotte, « Pauvreté d'Hector de Saint-Denys Garneau », p. 117.

${ }^{118}$ Poème « [Entre le ciel et l'eau] », CEurres, p. 116 ; Poìmes et Proses, p. 15.

${ }^{119}$ Extrait de «Noël — Impressions », CEuvres en prose, p. 56 ; Poèmes et Proses, p. 152.

${ }^{120}$ Lettres, p. 332.

${ }^{121}$ Poème « [Leur cœur est ailleurs] », CEuvres, p. 194 ; Poèmes et Proses, p. 54.

${ }^{122}$ CEures, pp.190-192. 